\title{
DIC Challenge: Developing Images and Guidelines for Evaluating Accuracy and Resolution of 2D Analyses
}

\author{
P. L. Reu ${ }^{1 \mathrm{a}}$, E. Toussaint ${ }^{\mathrm{b}}$, E. Jones ${ }^{\mathrm{a}}$, H. A. Bruck ${ }^{\mathrm{c}}$, M. Iadicola ${ }^{\mathrm{d}}$, R. Balcaen ${ }^{\mathrm{e}}$, D. Z. Turner ${ }^{\mathrm{a}}$, \\ T. Siebert ${ }^{\mathrm{f}}$, P. Lava ${ }^{\mathrm{g}}$, and M. Simonsen ${ }^{\mathrm{h}}$ \\ ${ }^{a}$ Sandia National Laboratory, Albuquerque NM, USA \\ ${ }^{b}$ University Clermont Auvergne, France \\ ${ }^{c}$ University of Maryland, USA \\ ${ }^{d}$ National Institute of Standards, Gaithersburg, MD, USA \\ ${ }^{e}$ KU Leuven, Ghent, Belgium \\ ${ }^{f}$ Dantec Dynamics GmbH, Ulm, Germany \\ ${ }^{g}$ MatchID, Ghent, Belgium \\ ${ }^{h}$ Correlated Solutions Inc., Columbia, SC, USA
}

\begin{abstract}
With the rapid spread in use of Digital Image Correlation (DIC) globally, it is important there be some standard methods of verifying and validating DIC codes. To this end, the DIC Challenge board was formed and is maintained under the auspices of the Society for Experimental Mechanics (SEM) and the international DIC society (iDICs). The goal of the DIC Board and the 2D-DIC Challenge is to supply a set of well-vetted sample images and a set of analysis guidelines for standardized reporting of 2D-DIC results from these sample images, as well as for comparing the inherent accuracy of different approaches and for providing users with a means of assessing their proper implementation. This document will outline the goals of the challenge, describe the image
\end{abstract}

\footnotetext{
${ }^{1}$ Corresponding Author: Email: plreu@sandia.gov, Tel. 505-284-8913, Fax. 505-844-6411
} 
sets that are available, and give a comparison between 12 commercial and academic 2D-DIC codes using two of the challenge image sets.

Keywords: Digital Image Correlation, DIC, metrology, full-field measurement

\section{Introduction - Description and goals of the challenge}

Digital image correlation (DIC) is a full-field image based method of measuring displacement and strains proposed and established for two-dimensional measurements in the 1980s [1-3], and for three-dimensional measurements in the 1990s [4,5]. For a full description of the algorithms and implementation of DIC we direct the reader to the seminal book by Sutton [6]. This paper only contains a brief review of DIC in a nontechnical context to orient a reader to the basic ideas of 2D-DIC. The paper then focuses on the DIC Challenge images, their creation and purpose, and a discussion of the results of the first DIC Challenge.

\subsection{Local DIC formulation}

The fundamental principle behind local DIC is to subdivide an image into small, individually resolved subsets (facets). The subsets are then each analyzed to measure the motion and deformation between integer pixel locations in the selected reference image and any deformed images. To resolve grey level values at sub-pixel locations in the deformed image, interpolation schemes are used to reconstruct the image. Most 2D-DIC software solves a nonlinear minimization function using the grey levels to find an optimum match. During this process the subset is allowed to deform by means of the subset shape function and the grey levels are interpolated for a subpixel match [7]. Various minimization formulations may be used to find the optimum match, for example the Sum of the Squared Differences (SSD) formulation (see the article series in Experimental Techniques [8] for a more complete outline of basic DIC methods from a practical viewpoint). The software then reports the found location of the subset in the deformed image. Added information may also be available at other inter-subset locations from the subset shape function, but is nearly always left unused. Many commercial code details are not documented or explained because of intellectual property concerns, although most employ the basic principle presented here. Consequently, many practitioners must use their software as a "black box". We direct the reader to the vendor's software documentation or papers published by the university codes when available for details about the implementation of specific software packages. 


\subsection{Global DIC Formulation}

Unlike local DIC, global DIC solves for the displacement field between the reference image and the deformed images for all points within the image simultaneously. To do this, the image is discretized with a finite element (FE) mesh, and then the displacement field is found by minimizing the grey level differences. The result is the nodal point motion. Results are found at other locations in the image using the FE shape functions. We direct the reader to Hild and Roux for more details on global DIC implementation [9]. Therefore, in principle, global and local DIC are very similar, except for the reconstruction approach to the deformed image and subsequent number of pixels used to correlate with the undeformed image.

\subsection{Design and Purpose of the DIC Challenge}

The DIC Challenge is in large part inspired by the Particle Image Velocimetry (PIV) challenge and is following a similar, although slower, course [10-12]. The goal of the DIC Challenge is not to decide which DIC code is best, but rather to provide a venue where codes can be verified, tested, vetted and discussed to help standardize analysis of their inherent accuracy and to help users properly employ them. Towards this end, the DIC Challenge Board carefully designed image sets to test problematic areas in DIC analysis. These include:

- Interpolation bias errors due to improper selection of interpolants.

- Inability to deal with sample rotation (as is the case in the frequency based methods).

- Robustness to image noise and/or lack of image contrast.

- Determination of the ideal balance between smoothing and spatial resolution.

The DIC Challenge images and accompanying analysis algorithms provide a standard for evaluating the accuracy and precision of DIC software. This toolbox has several applications:

1. Journal publications claiming improvements in the accuracy of DIC algorithms.

2. Investigation of commercial software to allow users to better understand the DIC "black box."

3. Code verification.

4. Comparisons between multiple DIC codes, for example local and global formulations.

An exercise like the DIC Challenge would not be complete without a "competition portion". For the competition, each commercial vendor or code-developer ran their own software using the provided sample images, which removed the possible problem of "inexpert" users. The vendors then reported their results to the DIC Challenge Board, and the results were analyzed using a common set of algorithms. We draw two main conclusions from this competition. First, while there are differences between the 2D codes in speed, ease-of-use, post-processing 
functions, etc., when run by an expert user, nearly all the codes analyzed for this paper returned essentially the same results. Second, the competition raised an important discussion that is a fundamental problem with DIC: the trade-off between spatial resolution and filtering of the data. A cautionary note is that users often prefer to have a smoother result, as it looks better to the human eye; however, this may not be the optimum result for a given use of the data. Both of these conclusions emphasize the need for training of DIC users.

\subsection{Important definitions}

Definitions are important when discussing measurement uncertainty. The DIC Challenge board follows the guidelines laid out in the Bureau International des Poids et Mesures (BIPM), an international body that helps define measurement standards and terminology. Two definitions from the BIPM vocabulary document are particularly important for this paper: Precision and Accuracy [13]. "Measurement Precision" is defined as the "closeness of agreement between indications or measured quantity values obtained by replicate measurements on the same or similar objects under specified conditions. "Measurement Accuracy" is defined as "closeness of agreement between a measured quantity value and a true quantity value of a measurand." In the context of this document, the measurand is the displacement in pixels at locations in the image. Strain is a derived quantity from the displacement measurand. "True quantity value" has a particular meaning and is often unknowable in practical situations. However, with synthetic images, we have a true value, and this is used in comparisons. "Resolution" is defined as "smallest change in a quantity being measured that causes a perceptible change in the corresponding indication." This term is used more colloquially in this document to indicate displacements that are measurable above the noise floor and is related to precision and the measurement noise in this sense. A final term "Spatial Resolution" is specific to DIC and is defined as the inverse of the cutoff frequency at some given decrease in signal.

\subsection{Paper content and organization}

The article is organized into three main sections. The first section briefly describes the image sets and the goals of the DIC challenge, including details on how we created the synthetic images and the relevant references. Appendix A contains example analysis of all the sample images and some typical DIC results obtained for them. The second section picks two image sets that the participants analyzed (Sample 14 and 15), describes the image sets and methods of analysis, and presents the results of the first challenge round. The final section in the paper 
presents conclusions and future goals of the DIC Challenge. The website includes information on the MatLAB and LabVIEW analysis codes ${ }^{2}$.

\section{DIC Challenge Image Sets}

Table 1 lists the DIC Challenge image sets available at the website https://sem.org/dic-challenge/ at the time of publication. We developed nineteen image sets, including rigid-body sub-pixel translations, rigid-body rotations, out-of-plane translation, spatially-varying displacement and strain gradients, and displacement discontinuities. Each image set seeks to challenge one or more characteristics of DIC analysis, such as interpolation bias, robustness to image noise, ability to account for rotations, and determination of the balance between spatial smoothing and spatial resolution. The numbering scheme of the image sets was arbitrarily created. However, we preserve this numbering scheme for existing journal articles that refer to this historical naming convention.

Image sets 1 - 13 and 16 - 17 serve two main purposes. First, the image generation processes were thoroughly vetted by a large cadre of DIC practitioners, which settled questions about the image creation and appropriateness for use in assessing DIC codes. Second, these image sets allow DIC code developers and users to verify ${ }^{3}$ and evaluate basic performance of their software. Towards this end, we provide the standard analysis algorithms for the results on the website. We also provide example analyses using a single, commercially-available DIC software in the supplementary information in Appendix A. Authors of publications describing new DIC algorithms or software are strongly encouraged to verify and evaluate their codes using these image sets and standard data analysis algorithms, with proper referencing, and present the results in their publication. The standard analysis algorithms also make comparisons between different codes straightforward. The use of standard images and analysis algorithms will provide an even playing field and a more easily reviewed paper when claims of 2D-DIC improvements are made.

For most well-implemented and fully-developed DIC codes, obtaining reasonably accurate results from samples $1-11,16-17$ is trivial. Therefore, two more image sets - Sample 14 and Sample 15 - were generated with the intention to challenge DIC capabilities, specifically probing the relationship between noise suppression and spatial

\footnotetext{
${ }^{2}$ Certain commercial equipment, instruments, or materials are identified in this paper in order to specify the experimental procedure adequately. Such identification is not intended to imply recommendation or endorsement by the National Institute of Standards and Technology, nor is it intended to imply that the materials or equipment identified are necessarily the best available for the purpose.

${ }^{3}$ Following the standard FE terminology, verification tests [14] the code to ensure that it is written correctly and is returning the correct answer.
} 
filtering. We discuss the analysis of these images by all participants of the DIC Challenge in the DIC Challenge Results section.

Table 1. Sample image set numbering and information. Image sets are available at: https://sem.org/dic-challenge/.

\begin{tabular}{|c|c|c|c|c|c|c|}
\hline Description & Set Name & $\begin{array}{l}\text { Method } \\
{[15,16]}\end{array}$ & Contrast & $\begin{array}{l}\text { Noise } \\
\sigma(G L)\end{array}$ & $\begin{array}{l}\text { Shift } \\
\text { (pixels) }\end{array}$ & $\begin{array}{l}\# \\
\text { Images }\end{array}$ \\
\hline TexGen Shift X,Y & Sample1 & TexGen & Varying & 1.5 & $X=Y=0.05$ & 20 \\
\hline TexGen Shift X,Y & Sample2 & TexGen & 0 to 50 & 8 & $X=Y=0.05$ & 20 \\
\hline FFT Shift X,Y & Sample3 & FFT Shift & 0 to 200 & 1.5 & $X=Y=0.1$ & 10 \\
\hline FFT Step Shift & Sample3b & FFT Shift & 0 to 200 & 1.5 & 0.05 to 0.5 & 5 \\
\hline FFT Shift $X, Y$ & Sample4 & FFT Shift & 0 to 50 & 8 & $X=Y=0.1$ & 10 \\
\hline FFT Shift X,Y & Sample5 & FFT Shift & Varying & 1.5 & $X=Y=0.1$ & 10 \\
\hline Prosilica Bin & Sample6 & Binning & 0 to 200 & 0.26 & $X=Y=0.1$ & 10 \\
\hline Prosilica Bin & Sample7 & Binning & 0 to 50 & 0.66 & $X=Y=0.1$ & 10 \\
\hline Rotation TexGen & Sample8 & TexGen & 0 to 100 & 2 & $\Theta$ by 1 & 10 \\
\hline Rotation FFT & Sample9 & FFT & 0 to 100 & 2 & $\Theta$ by 1 & 10 \\
\hline Strain Gradient & Sample10 & TexGen & 0 to 200 & 2 & Sinusoid & 10 \\
\hline Strain Gradient & Sample11 & TexGen & 60 to 130 & 2 & Sinusoid & 10 \\
\hline Strain Gradient & Sample11b & FFT & 0 to 200 & 1.5 & Tri. .01 to 1 & 6 \\
\hline Ex1 - Plate Hole & Sample12 & Exper. & Good & Low & $\mathrm{N} / \mathrm{A}$ & 12 \\
\hline Ex2 - Weld & Sample13 & Exper. & Poor & Low & N/A & 52 \\
\hline Varying Strain & Sample 14 & FFT & 0 to 200 & 5 & N/A & 4 \\
\hline Varying Strain & Sample 15 & TexGen & 80 to 180 & 2 & $\mathrm{~N} / \mathrm{A}$ & 9 \\
\hline Rigid Motion & Sample 16 & Exper. & 0 to 254 & 0.26 & $\approx 0.1$ & 11 \\
\hline Interpolant & Sample 17 & Exper. & & & & \\
\hline
\end{tabular}

\section{Image Generation Methods}

The images in the DIC Challenge were created by one of four means; two synthetic methods, one pseudoexperimental method and one experimental method. Synthetic images serve the purpose of providing a known solution against which DIC results can be compared, and their use is important for verification of the DIC software. Note that it is not possible to discover the experimental DIC uncertainty using only synthetic images. There are too many missing error sources and biases (e.g., spatial variations in illumination, pixel noise, and optical distortions) in the synthetic images to draw conclusions about any given experimental setup. Therefore, we also include pseudo-experimental and purely experimental image sets. While there are no known, exact solutions for these experimental image sets, results from different DIC codes and from a single DIC code run with different settings can be compared.

\subsection{Synthetic image generation}

The two methods of synthetic image generation used in the DIC challenge are the TexGen Software and the Fourier method. The TexGen software is documented in [16], which describes the mathematical approach used 
for creating "semi-realistic" images. TexGen is useful in that it uses analytical functions to define a speckle field which can then be sampled to create a deformed image. A drawback is that "real" speckle patterns cannot be used, making it impossible to evaluate problems that may be caused by the applied speckle characteristics. To work with experimental speckle patterns, we used a second method that exploits the properties of the Fourier transform expansion or the Fourier phase shift to create deformed or shifted images, as explained in [15]. Fourier methods have the advantage of allowing real speckle images to be used but use a different method of interpolation than that used by the DIC codes. In fact, Fourier shifting uses the ideal sinc interpolant function, but without having an infinite image size. The sinc function results in the most accurate interpolation possible, given the underlying data $[17,18]$. All other DIC software interpolants use a smaller support (region of the image) for the interpolant and are therefore some approximation of the ideal sinc interpolant. The bi-cubic spline is one well-known example used in many DIC codes. The DIC codes require this approximation for speed and spatial resolution considerations that do not occur when creating synthetic images. It is important to consider the influence of the synthetic image generation on the calculated results from DIC. This topic has been treated in [19] with the conclusion that the FFT and TexGen method can be used for determining what they term is the "ultimate error regime," i.e. errors caused only by the numerical DIC process interacting with image contrast and noise, but without other parasitic experimental errors. This is termed "verification" in this document.

Table 2 provides typical image noise for several types of machine vision cameras, as measured experimentally by acquiring five or more images of a completely stationary speckle pattern method. Completely stationary was obtained using an optical table and acquiring the images over only a few seconds. The pixel by pixel noise is then calculated and combined for the entire image to calculate the standard deviation of the grey values and the percent noise. Percent noise is calculated by dividing the measured noise standard deviation by the camera grey level range, 256 counts for an 8-bit image. For both the Fourier and TexGen synthetic images, image noise can be added to mimic camera noise. For these images, homoscedastic Gaussian noise was added of a given magnitude, as specified in Table 1. The image noise is specified as one standard deviation $(1 \sigma)$ of the grey level applied independently to each pixel. This is an approximation of the heteroscedastic noise profile of a typical machine vision camera [20]. Heteroscedastic noise scales with the pixel intensity, although still Gaussian in distribution. That is, brighter pixels have more noise than darker pixels in a generally linear fashion and can be measured using a grey card with a distribution of intensities and calculating the noise at the different intensities. Future simulations should take this fact into account. We assume that any discrepancies in the images between using a homoscedastic and heteroscedastic noise model will be small, although this remains unproven. 
Table 2. Typical machine vision camera noise levels

\begin{tabular}{l|l|l}
\hline Camera (8-bit) & \%Noise & StDev (counts) \\
\hline PointGrey GRAS (5MP) & 0.82 & 1.56 \\
\hline PointGrey GX (5MP) & 0.46 & 1.17 \\
\hline AVT Prosilica GX (5MP) & 1.7 & 4.1 \\
\hline Prosilica GE4900 (16MP) & 0.86 & 0.34 \\
\hline Prosilica Binned (0.1MP) & 0.1 & 0.28 \\
\hline Phantom v611 (1MP) & 0.78 & 1.84 \\
\hline Andor Neo (5MP) & 0.60 & 1.51 \\
\hline
\end{tabular}

\subsection{Pseudo-experimental image shifting}

We adopted a "super-resolution" approach as outlined in [15] to move closer to experimentally created images, but still with exact subpixel shifting. In brief, this technique uses a 16-Megapixel $\left(4872 \times 3248\right.$ pixels $\left.^{2}\right)$, highresolution camera with oversized speckles of 50 pixels. The image is then numerically binned, by averaging $10 \times 10$ "super-pixels" into a single pixel to create a lower resolution image of $487 \times 324$ pixels ${ }^{2}$. After numerical binning, the speckles are then a suitable size of 5 pixels. Then by changing the first binning row or column of the fullresolution image from index 0 to 9 , we achieve an exact subpixel shift of the binned pixel of 0.1 pixels. Pseudoexperimental shifting has the advantage of making no assumptions about how the camera hardware captures the image and it is therefore closer to an experimentally shifted image. Other researchers have adopted the superresolution image creation to minimize the influence of interpolation during synthetic image generation [21].

\subsection{Experimentally generated images}

Experimentally created images do not have the questions of authenticity that synthetic images have; however, knowing the "true value" for comparison with a DIC result is difficult or impossible. Experimental images have two uses: First, as a comparison between DIC codes or different settings within the same code. And second, as a comparison with a ground truth secondary measurement as in Sample 16 with rigid body motion, where the position is known to subpixel accuracy using an optical encoder on the stage.

\subsection{Other image details}

All images are monochrome 8-bit tagged image file format (*.tif or *.tiff). We chose 8-bit images because it has been shown that higher bit count does not noticeably improve the DIC results [22,23], therefore results using 10 bit or higher images would not change any conclusions. Rather, the magnitude of the matching error is proportional to the ratio of image contrast to image noise [23]. The DIC Challenge explores this relationship in all the image sets by varying the image contrast and the noise level. In practice, the connection between the noise and contrast also scales with camera bit depth, lessening the advantage of greater than 8-bits for DIC, all other 
experimental parameters being equal (ignoring some experimental niceties). Each sample directory provides either a comma separated text file (*.csv) or spreadsheet file that contains information pertinent to the image set, whether that be the shifting or deformation profiles or the displacement is encoded in the image filename. This information provides the user with the known solution, against which DIC results can be compared for code verification.

\section{Description of Image Sets for Code Verification}

This section describes image sets 1-12 and 16-17 provided for basic DIC code verification. Analysis of these sample images are in Appendix A with a brief discussion of the expected results. Standardized analysis algorithms are provided on the website.

\subsection{Sample 1 and Sample 5 (Shifted image with varying contrast using TexGen and FFT)}

Sample 1, and 5 are both rigid body shifted images with constant noise (standard deviation of 1.5 counts grey level in an 8-bit image) and varying contrast. We created Sample 1 using TexGen and Sample 5 using the Fourier method. The images are shifted in both $x$ - and $y$-directions simultaneously in increments of 0.05 pixels/step (Sample 1 - see Appendix A, Figure 17) and 0.1 pixels/step (Sample 5 - see Appendix A, Figure 18). Figure 1 shows the first and last image histogram for Sample 1. Sample 1 provides a varying contrast throughout the image series. Most DIC codes cannot correlate the entire series unless a Zero-Normalized Sum of Squared Differences (ZNSSD) algorithm is used because the contrast shift is different for each image. It is important for DIC codes to compensate for contrast changes over time because in many DIC experiments, particularly for stereo-DIC, there will be changes in the contrast during the experiment.
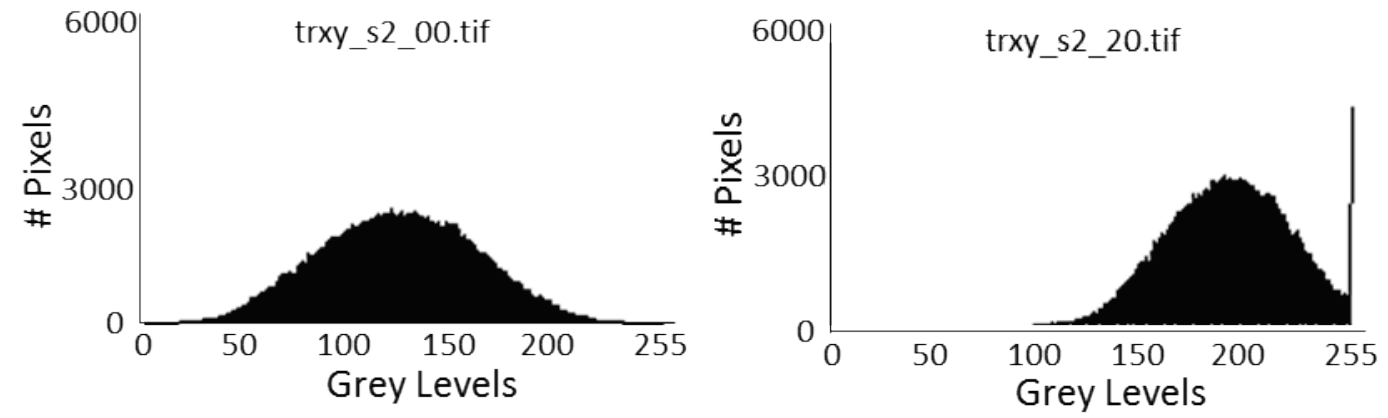

Figure 1. Histograms for first and last image of Sample 1 showing the change in contrast and saturated pixels for image trxy_s2_20.tif. 


\subsection{Sample 2 and Sample 4 (Shifted images with extremely high noise using TexGen and FFT)}

Another significant challenge for DIC is noisy images. Noisy images are not common with traditional machine vision cameras, but can be prevalent in alternative imaging methods, including x-ray, atomic force microscope, scanning electron microscope, ultra-high-speed cameras, and so forth. For example, the Sample 2 and Sample 4 image sets have an applied noise with a standard deviation of 8 counts and a fixed contrast spread of 50 counts. Sample 2 was created using TexGen, and Sample 4 using the Fourier method. Figure 2 shows just how poor these images are, with the noise being obvious in the image.

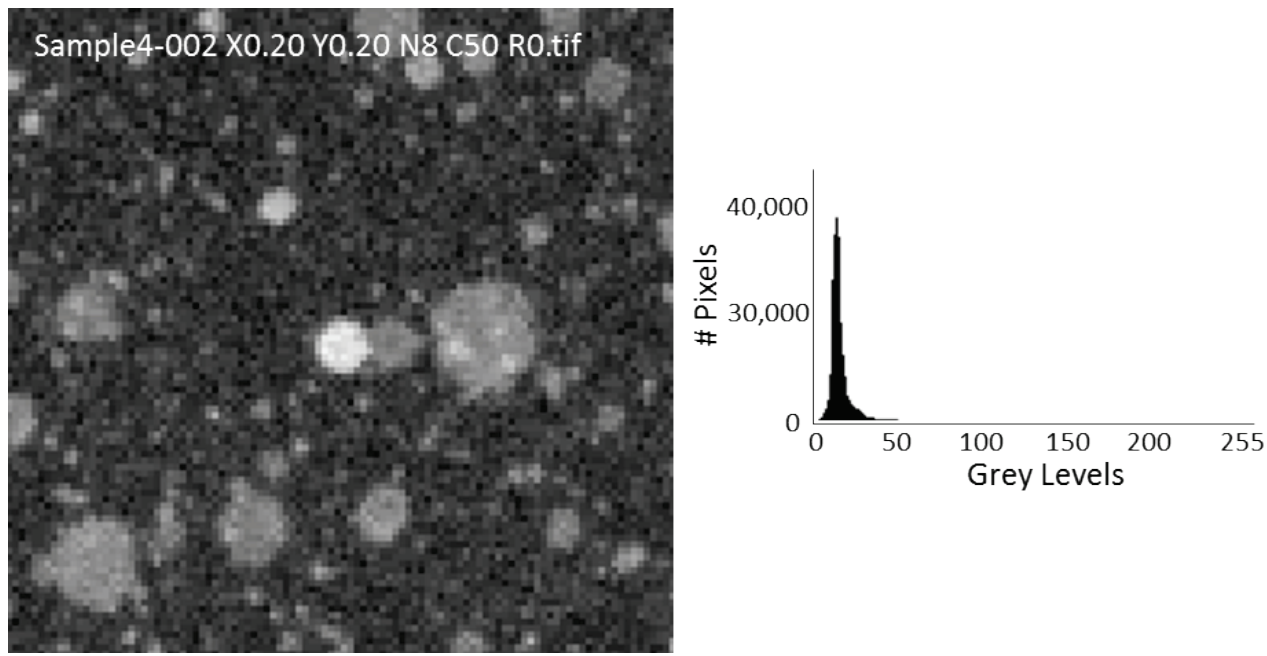

Figure 2. Histogram equalized plot of a small region of the image showing the obvious noise and the speckles. Corresponding histogram shows the small range of contrast available for matching by the DIC algorithm.

\subsection{Sample 3 and Sample $3 b$ (FFT Shifted image)}

Sample 3 and Sample $3 \mathrm{~b}$ are examples of good contrast and low noise images shifted using the FFT function. Sample 3 is a rigid body shift of 0.1 pixels per image and will nicely reveal both the DIC interpolation bias and variance. Sample $3 b$ uses the same FFT shift, however, only the bottom half of the image is shifted. Sample $3 b$ can be used to look at the effect of discontinuities in the results and the roll-off of the DIC filtering by applying a step function.

\subsection{Sample 6 and Sample 7 (Pseudo-experimental rigid body shift with low and high noise floot)}

Sample 6 and Sample 7 were created using a 16-Megapixel ultrahigh resolution camera image and the superresolution approach discussed in [15]. Sample 6 represents a high-contrast-low-noise image created experimentally and shifted via binning. This is as close to a completely experimental, perfectly-subpixel shifted 
image as is possible. The binning was the only numerical processing done; neither the contrast nor the noise was changed. Typical noise levels for machine vision cameras are 1 to 2 counts, as shown in Table 2 . The noise present in the image of Sample 6 of 0.26 counts is low for a machine vision camera because of the 10-pixel binning which suppresses the noise by a factor of $1 /$ sqrt $(N)$, where $N$ is the number of binned pixels. Therefore, in general this is a lower image noise than would be encountered in a traditional non-binned machine vision camera. Sample 7 has a higher noise floor of 0.66 counts, which we experimentally obtained by increasing the gain of the camera to increase the image noise rather than adding noise in after the binning. The unbinned image had 5.5 counts of noise. The binning procedure used in Sample 7, even with a high gain on the raw image, results in an image noise that is still modest for a machine vision camera. Additionally, the contrast range in the experimental image was sub-optimal in Sample 7 to exacerbate any matching errors in the DIC analysis.

\subsection{Sample 8 and Sample 9 (Rigid body rotation using TexGen and FFT)}

Samples 8 and 9 were created to test a potential flaw in DIC codes in how they deal with rigid body rotation. In early codes, inaccurate displacements caused by image rotations were inherent in the simple frequency based cross-correlation methods used. In spatial-minimized codes, problems with the shape function may also result that create bias errors when the sample rotates. We created Sample 8 using the TexGen software and Sample 9 using an FFT rotation method [24]. Both samples are a rigid body rotation from $0^{\circ}$ to $10^{\circ}$ in $1^{\circ}$ increments about the image center without any deformation. Both images have approximately 100 counts of contrast and an image noise of 2 counts $(1 \sigma)$.

\subsection{Samples 10, 11 and 11 b (Simple strain gradients using TexGen and FFT)}

Samples 10,11 and $11 \mathrm{~b}$ were created to have a simple strain gradient variation across the sample. Samples 10 and 11 were produced with TexGen using the quasi-sinusoidal function from Fazzini [25]. The 10 images have increasing magnitudes of the same function. The two samples are different in that the contrast range for Sample 11 is only 70 counts while Sample 10 has 200 counts.

Sample $11 \mathrm{~b}$ is a triangle displacement field created using a Fourier expansion by 100 and then a linear interpolation to find the grey value at the shifted pixel location. This creates a discontinuity in the strain field, which helps to probe the spatial resolution of the DIC package. In signal processing terms, this is equivalent to putting a strain level step function into the DIC "filter" and looking at the roll-off. 


\subsection{Sample 12 and Sample 13 (Experimental results from a tensile test on a hold and weld)}

Sample 12 is a purely experimental data set using a painted speckle pattern on a tensile specimen with a hole in the middle. A publication by Passieux [26] used these images and more details are available there. As this is an experimental image series, there are no ground truth data for comparison with the DIC results. This data is useful for virtual strain gage size studies into the spatial resolution and noise robustness of DIC codes, as well as roundrobin type tests for comparing one code versus another code of different DIC formulations (global versus local for example).

Sample 13 is included as an example of more "difficult" experimental imaging conditions. The data is from a 2D experiment using a ring light and bead-blasted surface preparation. The purpose of the experiment was to characterize laser welds in 304L stainless steel [27]. The advantage of using the bead-blast created surface contrast in the experiment is there was no paint debonding at the high strain values. This choice however introduces a problem of "evolving" speckles during the test. It is therefore difficult to run this analysis without using incremental correlation. A larger number of images (52) is provided to minimize the difference in speckle appearance between any two images in the series. The speckles are also most likely aliased, which introduces another error source and a particular challenge for certain interpolants (e.g. bilinear). However, the strains are large providing a good signal to noise ratio. The weld is near the middle of the sample, as indicated by a small black line on the right edge of the sample as labeled in Figure 3.

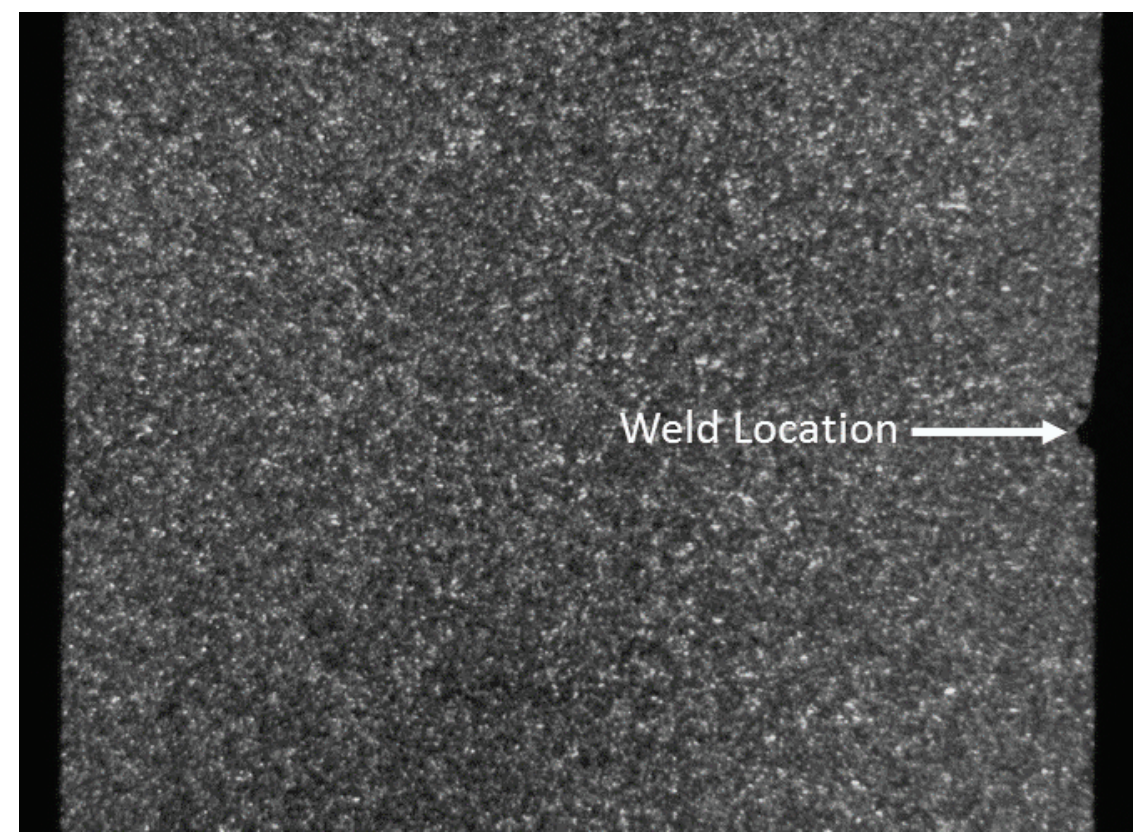

Figure 3. Reference image from Sample 13 showing the questionable speckle quality and the weld location. Speckles are created by using a ring light on a bead blasted surface. 


\subsection{Sample 16 (Experimental results for in-plane, rigid body translation)}

To explore interpolation error, we acquired "exact" subpixel shifted experimental images using an Aerotech nmprecision displacement stage and a Prosilica 16-MPixel camera with a Nikon 28-85 mm zoom macro lens (see Figure 4). The speckle pattern was approximately 50 pixels in the full resolution image. The image was then decimated by a factor of 10 as described in [15] to yield speckles that were roughly 5 pixels in size. Note that the sample was shifted mechanically by moving the stage, rather than the pseudo-experimental method discussed previously. The binning was used purely to decrease the image noise. The binning approach has two benefits: First, it makes the stage step size $10 \times$ larger on the object, improving the displacement resolution of the stage by a factor of 10. Second, it improves the noise profile of the camera from $\sigma_{\text {counts }}=0.86$ grey levels down to about $\sigma_{\text {counts }}=0.26$ gray levels. To improve the noise even more, 5 images at each stage position were averaged, after first being binned, to yield a single image for analysis. We moved the stage in 33.5 micron steps, which corresponded to 0.1 pixels per step, for 10 steps. The stage encoder measured the true position at each step to have a secondary "known" measurement of stage motion against which DIC results can be compared. The vendor calibrated stage had an absolute position accuracy of $\pm 280 \mathrm{~nm}$ over the entire 160 -mm travel range, which corresponds to $+/-0.0008$ pixels. The vendor stated position resolution should be even better over the $0.33 \mathrm{~mm}$ travel used for this subpixel shift.

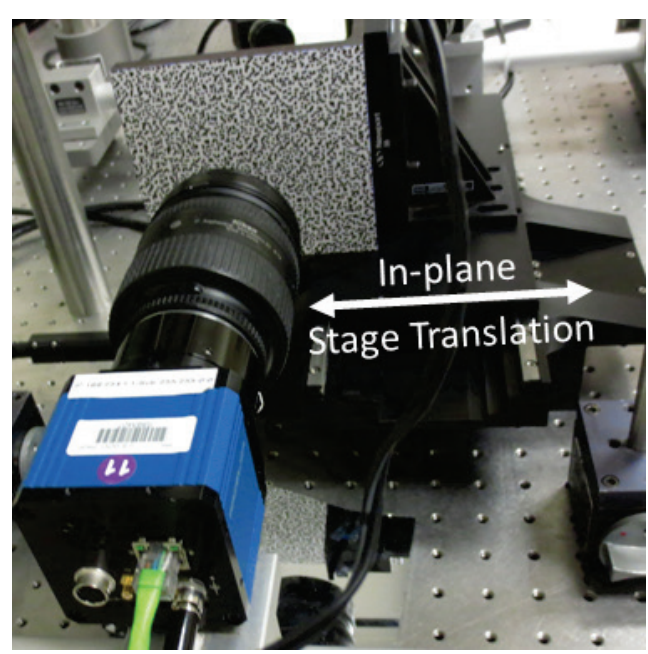

Figure 4. Experimental setup for Sample 16. Prosilica 16 Megapixel camera and Aerotech stage used for in plane translation.

\subsection{Sample 17 (Experimental results for out-of-plane rigid-body translation)}


A second, cheaper and simpler approach to explore interpolation error was designed, and the experimental setup is presented in Figure 5 (compare this with the experimental setup used for Sample 16). A speckle pattern adhered to a manual linear stage was translated out-of-plane, perpendicular to the camera axis. Out-of-plane motion towards the camera of several millimeters generates effective sub-pixel in-plane expansion. Therefore, a simple manual stage has sufficient displacement resolution to provide accurate "known" applied displacements. This simple setup can be used to probe the quality of the interpolant and to discover the magnitude of interpolation errors relative to variance errors. While experimental data is available on the DIC Challenge website, this tabletop experiment can easily be replicated. The Field of View (FOV) was roughly 100-mm with a Point Grey 5Megapixel Grasshopper camera and an Edmund Optic 35-mm lens.

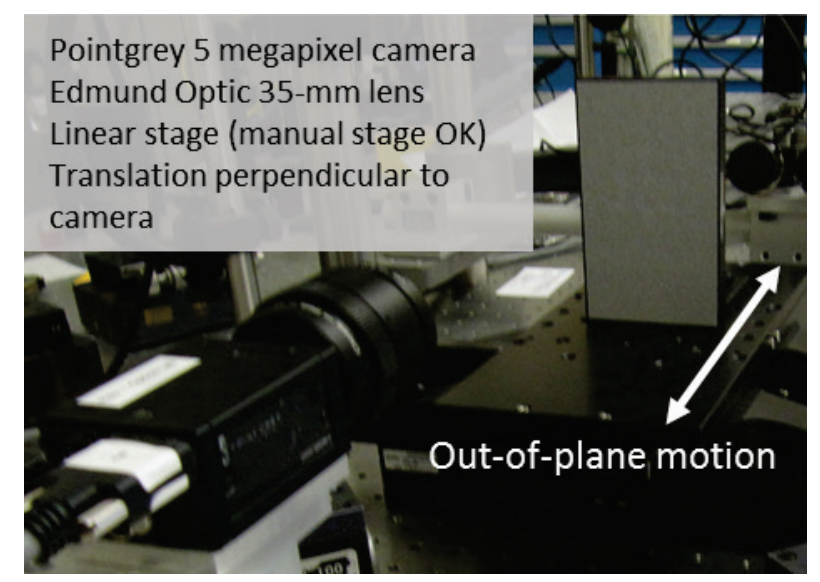

Figure 5. Sample 17 out-of-plane translation experiment. Note that a manual stage will work as well. Floating optical table is not required.

\section{DIC Challenge Results}

\subsection{Selection of images for challenge participants}

After thorough vetting of the image generation process by the participants using Samples 1 through 11, Samples 14 and 15 were generated and provided for analysis to the challenge participants. In this round of the Challenge, participants were also provided with the exact solution. These images were designed to probe the connection between displacement and strain noise suppression and spatial filtering in the DIC analysis, a balance that every DIC user must make in every DIC analysis. The participants were only allowed to submit one set of results for analysis. A future blind study may include the possibility to provide two different results to illustrate the trade-off between spatial resolution and filtering within one DIC formulation. 
For this preliminary study, 9 participants elected to provide results to the DIC Challenge board. They included five commercial vendors (all existing vendors at the time), three university codes and two global codes. The results were submitted as a text based delimited file with the displacements and strains reported at a 5-pixel spacing over the entire image area. Table 3 lists the participants, type of code and name of the 2D-DIC software used for the analysis. We do not reveal the correspondence of the codes/vendors and the results in this paper. We desire in a future blind study that includes a broader cross-section of participants to include the names of the participants with the results. However, at this point in the Challenge, anonymity was useful to encourage participation, mitigate fears, and allow for open discussion on how to "score" the images.

To score the quality of the results is a difficult task, fraught with the dual challenge of analyzing the entirety of the full-field results, while simultaneously deciding metrics on which to score them. This task is even more challenging when using a single set of results to determine quality: Does the participant choose to reduce noise, but at the expense of increasing bias errors, or try to capture the peak, but at the expense of increased noise? The answer to the question of balancing noise and bias error with spatial resolution is dependent on the data use. Therefore, "scoring" will always be subjective. However, this preliminary study did prompt discussion and an understanding of how to quantify the spatial resolution of DIC. This paper seeks to disseminate that information to a wider audience to help provide a means for evaluating DIC software in other journal publications.

Table 3. List of DIC participants

\begin{tabular}{|c|c|c|}
\hline Participant & Code Description & Code Type \\
\hline Elizabeth Jones & MatLAB Code & Local, subset based \\
\hline Stephane Roux/François Hild & $\begin{array}{l}\text { Previously a university code } \\
\text { http://www.correli-stc.com/ }\end{array}$ & Correli $^{\mathrm{STC}}-$ Global \\
\hline Daniel Turner & $\begin{array}{l}\text { Sandia National Laboratory Code } \\
\text { https://dice.sandia.gov/ }\end{array}$ & $\begin{array}{l}\text { DICe - Global } \\
\text { DICe - Local }\end{array}$ \\
\hline Pascal Lava/Lukas Wittevrongel & $\begin{array}{l}\text { Previous university code } \\
\text { http://www.matchidmbc.com/ }\end{array}$ & $\begin{array}{l}\text { MatchID - Local } \\
\text { AdaptID - Global }\end{array}$ \\
\hline Micah Simonsen & $\begin{array}{l}\text { Correlated Solutions Commercial Code } \\
\text { http://correlatedsolutions.com/ }\end{array}$ & Vic2D - Local \\
\hline Thorsten Siebert & $\begin{array}{l}\text { Dantec Dynamics Commercial Code } \\
\text { http://www.dantecdynamics.com/ }\end{array}$ & Q-400 Istra4D \\
\hline Bernd Wieneke & $\begin{array}{l}\text { LaVision Commercial Code } \\
\text { http://www.lavision.de/ }\end{array}$ & StrainMaster DIC \\
\hline Markus Klein/Tim Schmidt & $\begin{array}{l}\text { GOM and Trilion Commercial Code } \\
\text { http://www.gom.com/ }\end{array}$ & ARAMIS \\
\hline Kyle Lynch & Personal Code & PIV based code \\
\hline
\end{tabular}


Remember: The goal of the Challenge is to provide a venue where the DIC community can come to compare and improve their DIC codes and discuss critical topics such as "spatial resolution" and how to quantify and to understand the DIC results. We do not seek to create a hierarchy of DIC codes. The images will be available for future developers and users of DIC code who utilize these standard images to test their code and learn about using DIC software.

\subsection{More detailed description of image sets provided to participants}

\subsubsection{Sample 14 (Spatially varying sinusoidal displacement field)}

Sample 14 was created using a standard FFT expansion of the image by a factor of 100 . This super-resolution image was then linearly interpolated to find the grey value at the subpixel location, using a displacement field, $U_{x}(x)$, defined by the function in Eq. 1 .

$$
U_{x}(x)=2 \pi \alpha \cos \left(\frac{2 \pi x}{p}\right)
$$

Equation 1

where $p$ and $\alpha$ are the relative period and the amplitude, which can be varied to create a varying frequency and constant amplitude sine wave. The images were created with a constant displacement amplitude indicated in the filename and results file; however, this creates a varying strain amplitude throughout the image (see Figure 6). Future image sets will include both constant strain amplitude and constant displacement amplitude images to explore the filtering trade-off in both spaces separately and equally.

Several different noise levels were explored during the development of the images, including noise varying between 0 through 5 counts. While 5 -counts of noise is high for a machine vision camera (between 1 and 2 counts is more typical, c.f. Table 2), we chose the higher level to better explore the key trade-off between spatial filtering and noise in the displacement and strain fields. A noisier image will worsen the displacement and strain noise, requiring the user to select a balance between more filtered results (for example with a larger subset or strain window) versus better spatial resolution.

Note that this function is in an Eulerian coordinate system and needs to be corrected to a Lagrangian coordinate system for comparison with the DIC results. This detail is true both of the FFT Sample 14 images and Sample 15 TexGen images, and is discussed in Section Euler versus Lagrange. 


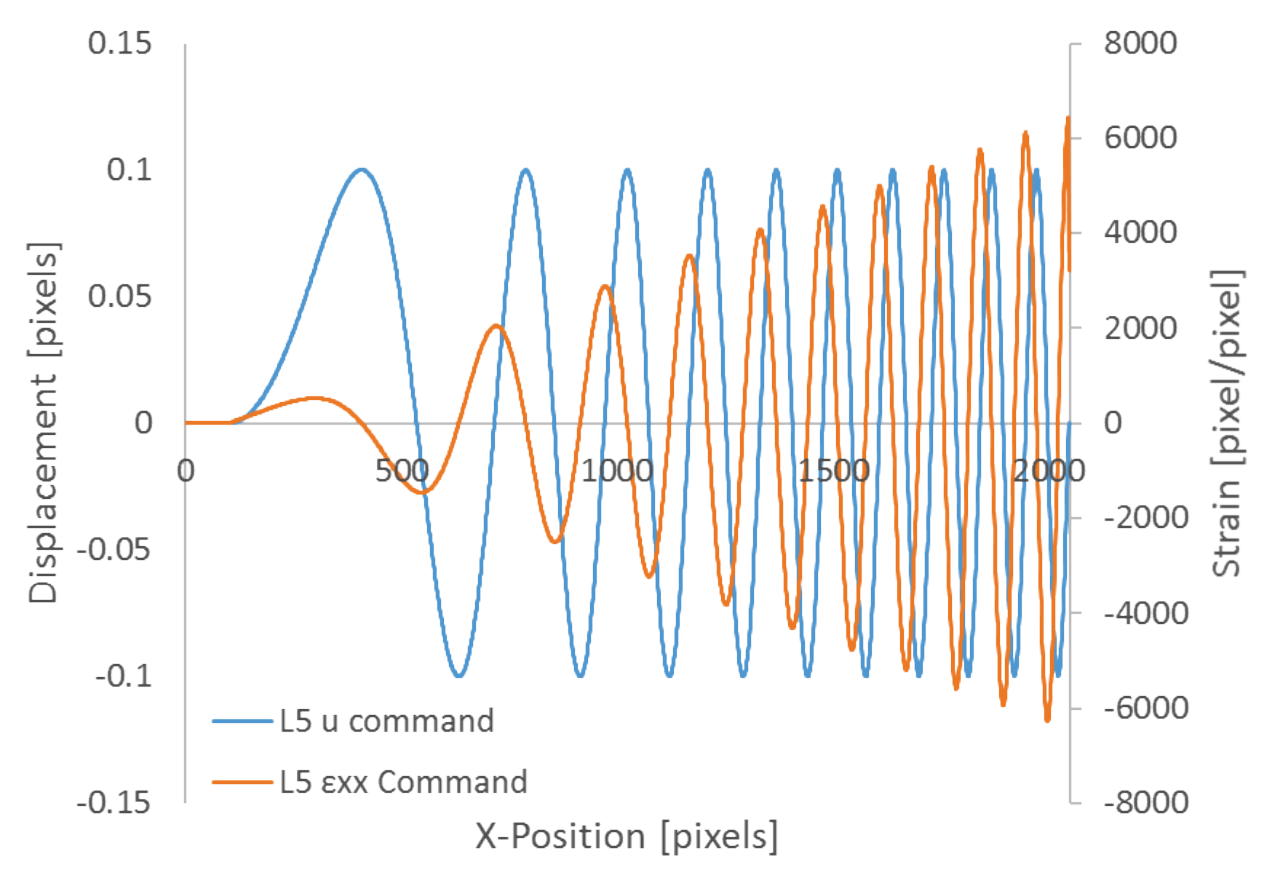

Figure 6. Commanded displacement and strain field for "Sample 14 L5 Amp0.1.tif". Commanded displacements and strains are identical for all $x$-rows in the image.

\subsubsection{Sample 15 (Spatial varying displacement field with discontinuities)}

Sample 15 complements the results from Sample 14 by providing a displacement field with sharp discontinuities. This tests the spatial resolution similarly to many cases found in common DIC experiments, for example, grain boundaries, material cracks, etc. Sample 15 was created using the TexGen software. While Sample 14 used a real experimental image as the basis, Sample 15 is a synthetically generated image. Sample 15 varies the displacement in the vertical direction. The displacement profile is available at the pixel locations in the associated data file. Figure 7 shows an example of the strain profile from the results for both the full-field results and the line cut profile of the displacement and strain. 


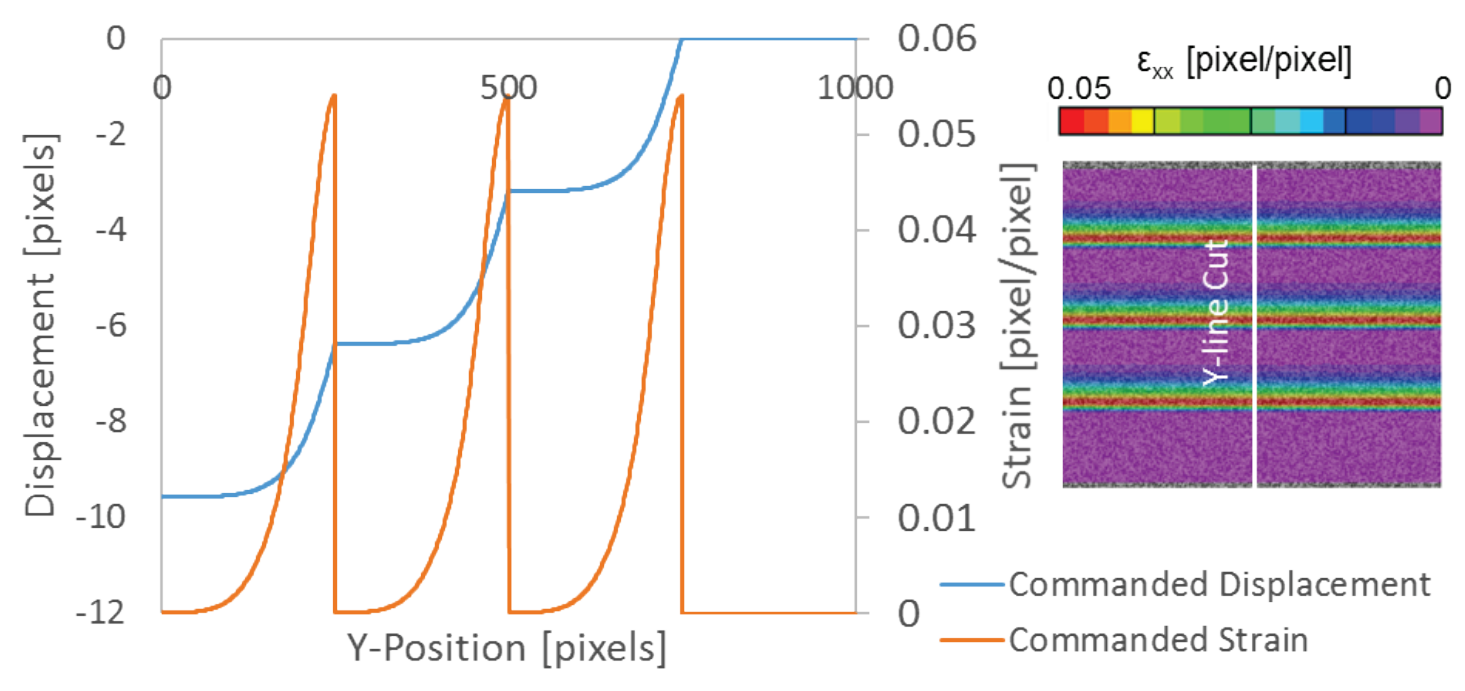

Figure 7. Sample 15 k250 commanded displacement and strain field. Commanded strain is identical for all y-columns in the image. Color plot is the full-field strain $\varepsilon_{x x}$.

\subsection{Euler versus Lagrange: An important detail}

Both Sample 14 and Sample 15 were plagued in the early rounds of the challenge with having the "measured" DIC results and the "known" synthetic displacement field in different coordinate systems (i.e., the commanded displacements are naturally in Eulerian coordinates, while the DIC results are naturally in Lagrangian coordinates). An accurate analysis requires the transformation into a common coordinate system. The coordinate system issue is an important but subtle point in image generation, and stresses the need for a well-vetted set of standard images. To the authors' knowledge, this article is the first to notice and correct this issue.

The error associated with the Eulerian and Lagrangian frames can be a difficult idea to explain and illustrate, but we try to do so here. First, two concepts: DIC is inherently Lagrangian in that it tracks the subset from the reference location in the reference image to its new location in the deformed image. The subset is the boat floating down the river in the famous example. Second, simulations are naturally Eulerian. That is, the speckles (effectively intensities or grey values in the image) are moved to a new position by applying the desired displacement field to the image intensity values. The intensity values at the fixed pixels in the deformed image are then calculated from the shifted speckle pattern. The pixels are like standing on the bridge looking down on the speckle field floating underneath. We illustrate the difference between Lagrangian and Eulerian description of motion in Figure 8, which shows a speckle pattern that was shifted by a linear, horizontal stretch. The speckles associated with region " $F$ " are moved to the new location by applying the deformation field (blue line). The rectangles correspond to pixels 
(as this is an illustration ignore that the pattern is too small), and the new grey values are calculated at these fixed pixel locations as the speckle field translates under the pixels. This makes the simulation inherently Eulerian.

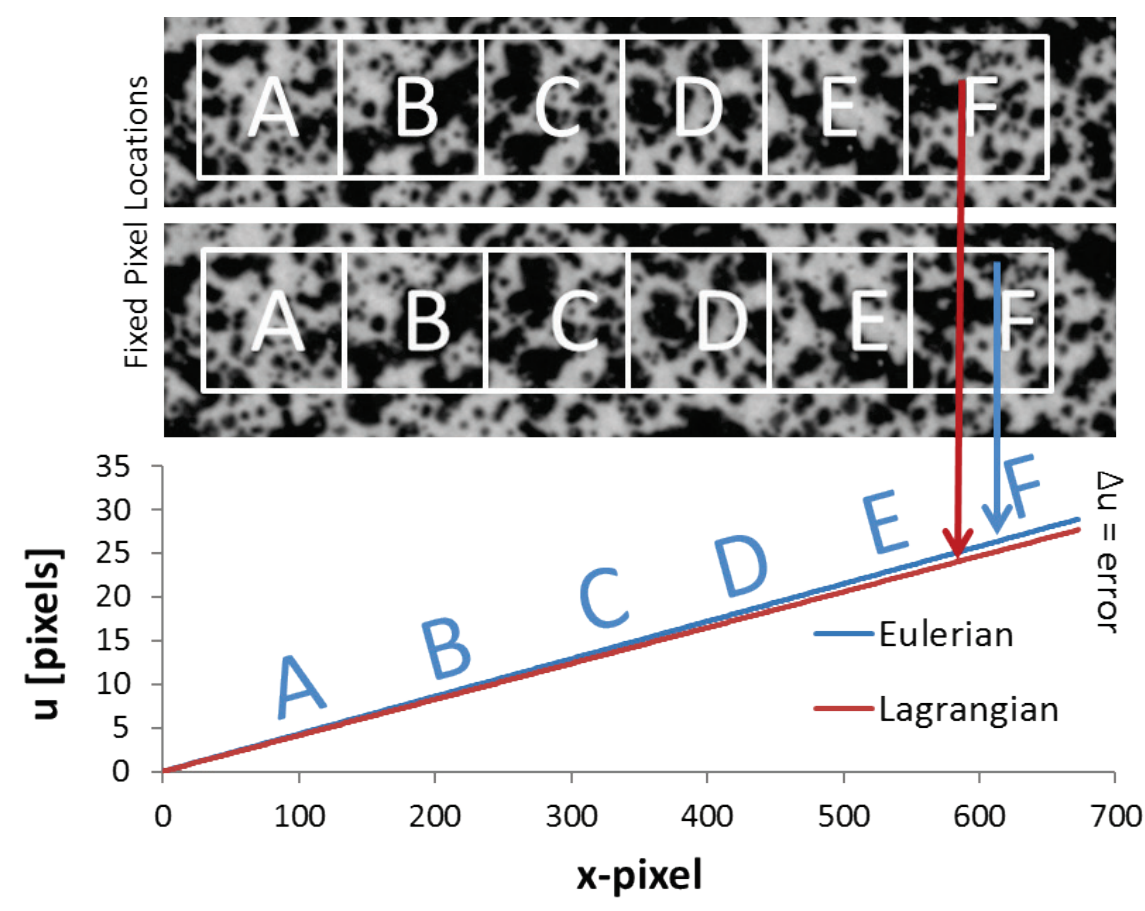

Figure 8. Illustration of the Lagrangian versus Eulerian problem.

There are two possible fixes to the results. The first involves using the function used to create the displacement and strain field, and finding the displacement value at the correct Lagrangian location $\left(X_{L}\right.$ and $\left.Y_{L}\right)$, found by getting the displacement at $X_{L}=\left(X_{E}-u\left(X_{E}\right)\right)$ or $Y_{L}=\left(Y_{E}-v\left(X_{E}\right)\right)$; where $X_{E}$ and $Y_{E}$ represent the integer pixel locations in the reference Eulerian state and $u\left(X_{E}\right)$ and $v\left(Y_{E}\right)$ the commanded displacements from that reference state. Note that these coordinates will be at a non-integer subpixel positions, whereas the commanded $u$ and $v$ values calculated were at reference integer pixel locations in the Eulerian coordinates. We used a second solution that involves interpolating the command position to find the correct value in the Lagrange coordinates for comparison with DIC. The same equations are used to find the $X_{L}$ and $Y_{L}$ locations at which the commanded displacement field is then interpolated. A cubic spline interpolant was used for Sample 14 and a Hermite polynomial (pchip in MatLAB and an equivalent LabVIEW function) was used for Sample 15 as it deals with the discontinuity better. Figure 9 shows the effect of this error on the results for a 1D cut through the data in the middle of the image of Sample 14. Note that looking at the curves for $u_{E}$ versus $u_{L}$ it is not possible to tell the difference between the two results, and indeed for these curves the error between the two coordinate systems is less than $3 / 1000^{\text {th }}$ of a pixel. This is well below experimental errors; however, synthetic images must be correct! Errors in Sample 15 were similar but of larger magnitude because of the larger displacements involved. 


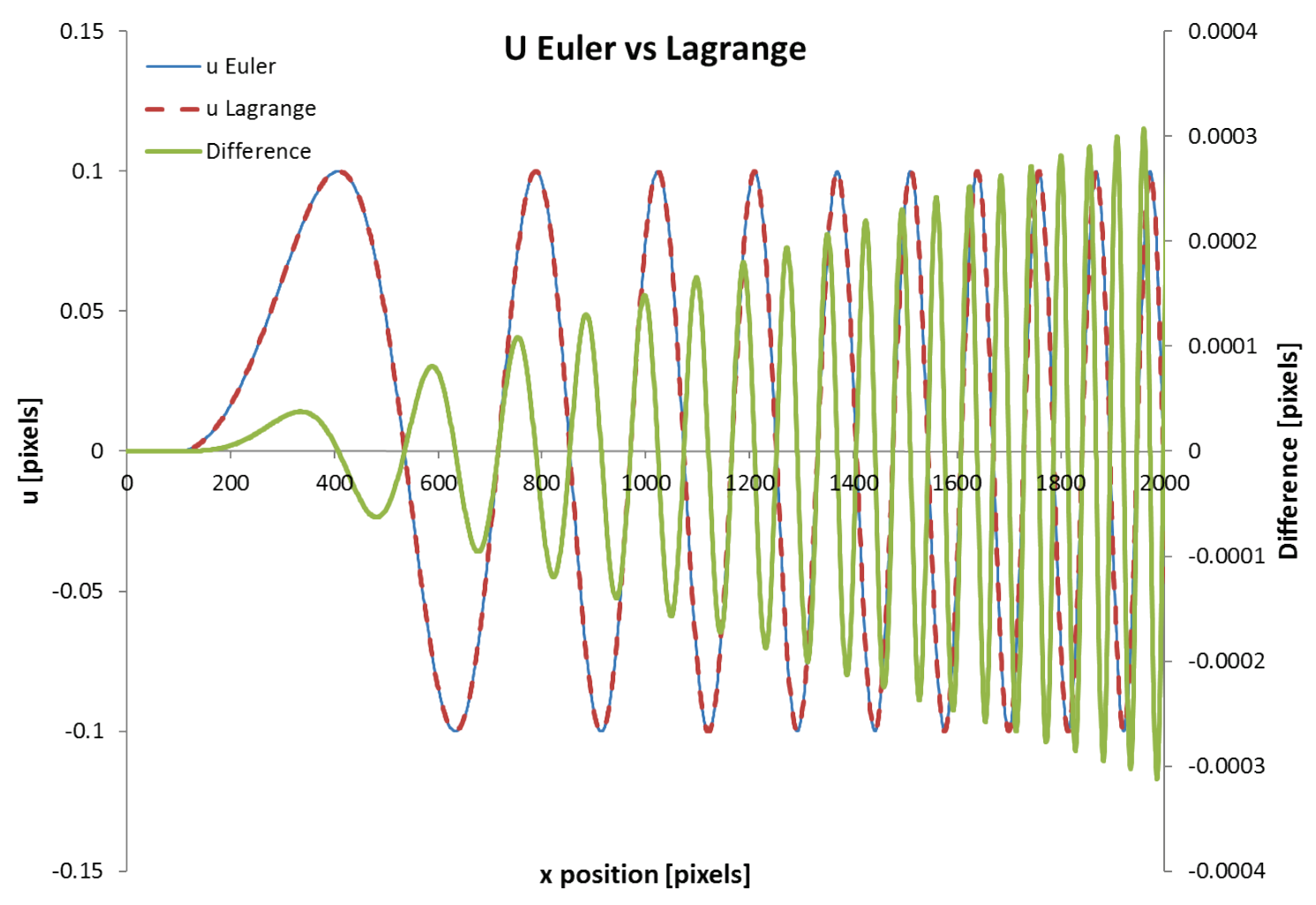

Figure 9. Illustration of the Euler versus Lagrange error using Sample 14. Blue curve is the commanded displacement field, red curve is the corrected Lagrange displacement field (aka the DIC results) and the green curve is the difference. Note the growing error as it accumulates across the image.

\subsection{Sample analysis procedure}

\subsubsection{Analysis Software}

The analysis presented in this paper was done using National Instruments LabVIEW software (v2015), and the results were verified using MatLAB code written by a second person. The executable files and source-code for both the LabVIEW and MatLAB analysis codes are available for download at the DIC Challenge website. DIC researchers are encouraged to make use of these codes as suitable in publications claiming 2D-DIC accuracy improvements, and DIC users are encouraged to use these codes to understand the "black box" that is most commercial DIC software. A standard set of algorithms, used by all authors, makes comparisons of different DIC algorithms and software packages more straightforward and efficient.

The analysis code uses standard array definitions of the image processing community with index $(0,0)$ defined as the top-left corner of the image, and rows and columns defined as in Figure 10. Standard computer numbering for arrays is also used, with numbering starting at 0 rather than 1 . Note: some of the submissions were off by one 
pixel and were shifted accordingly. The directions for the displacements and strains are also defined in Figure 10 using standard DIC notation.

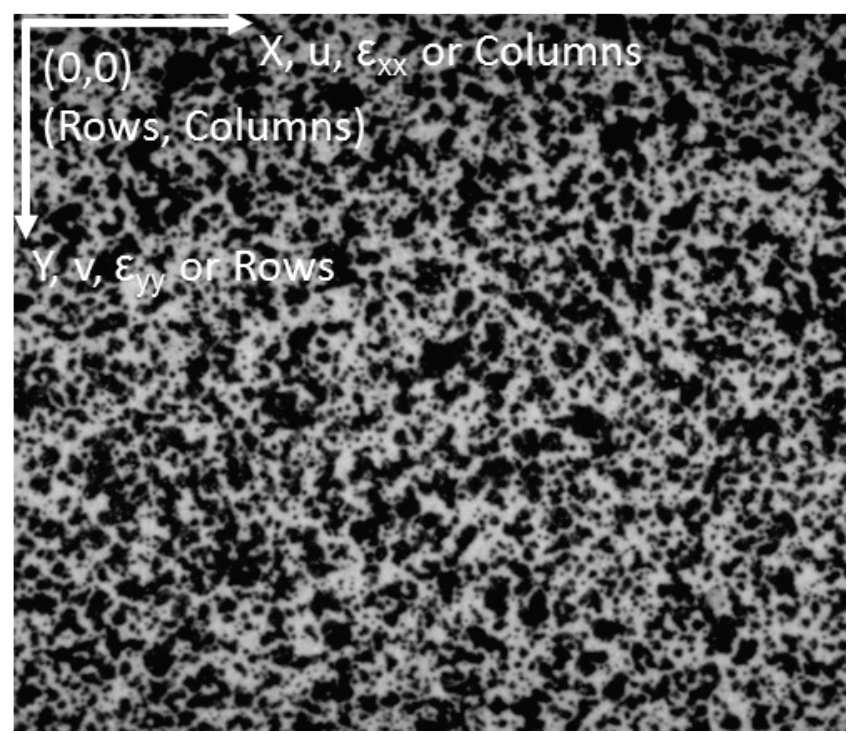

Figure 10. Coordinate system definition for the analysis codes. We use the standard "Image" definition for arrays, with 0,0 at the top-left corner of the array.

\subsubsection{Submission Guidelines}

The results were submitted using a comma separated value file (*.csv). We created the LabVIEW and MatLAB code with the flexibility to deal with the inevitable differences in the file format between the US, Europe and Asia. Separators in the file can include either commas (US), semicolons (Europe), or tabs. Flexibility was also incorporated in the code to define the decimal delimiter as either a period (US) or a comma (Europe). Participants reported the results with a 5-pixel step size in both the rows and columns. Global results reported the value at the center of the pixel to match the results of the subsets. Note that any errors of a possible $1 / 2$-pixel discrepancy due to these definitions should be small relative to the other errors encountered. The 5-pixel step size in result reporting, however, led to significant difficulties in precise analysis as data interpolation between the reported values was required. We describe this interpolation in detail in the results section. Future results will be submitted with a 1-pixel step in the results to simplify and improve the analysis.

\subsubsection{Line Cut Analysis}


Both Sample 14 and Sample 15 were 1D displacement fields imposed on 2D images. Therefore, each row in Sample 14 and column in Sample 15 have the same displacement and strain. This data redundancy was used to probe the bias and noise errors of DIC by averaging along either the rows or columns. Doing this yields a mean value, which provides a cleaner visualization of the bias errors. Bias errors for the synthetic images indicates an offset from the known value and is caused by spatial filtering of the DIC algorithms, by the subset size and shape function, and by the virtual strain gage used to calculate the strain. The standard deviation at each point along the row or column indicates the noise. All plots and figures unless otherwise stated use 1 -standard deviation $(1 \sigma)$ for the reported noise value.

\subsubsection{Root Mean Square Error}

Another approach used to summarize the error of the full-field results in a single number is the Root-Mean Square Error (RMSE). Bornert [28] introduced this concept in his DIC uncertainty paper. The following equations define the RMSE for the displacement $u$ (or $v$ ), and similarly, but not shown, for the strain $\varepsilon_{x x}$ (or $\varepsilon_{y y}$ ).

$$
\begin{gathered}
R M S E=\sqrt{\frac{1}{n} \sum_{i, j}[\Delta u]^{2}} \\
\Delta u=u_{i, j}^{\text {Commanded }}-u_{i, j}^{D I C}
\end{gathered}
$$

Equation 3

where $i, j$ represent the row and column pixel locations and $n$ is the total number of reported DIC data points. Because subset-based codes do not provide data at the image perimeter (and because the size of this region without data varies for different DIC codes), we calculated the RMSE for Sample 14 only in the interior region from pixel $(50,50)$ to $(545,1995)$ in an image size of $588 \times 2048$ for 39,000 data points. Similarly, for Sample 15 , we used the interior region of $(50,50)$ to $(945,1930)$ in an image size of $1000 \times 2000$ for 67,304 data points.

\subsubsection{Spatial Resolution Measurement}

DIC can be thought of as a nonlinear low pass filter in the spatial domain. The cut-off frequency and roll-off of the filter are determined by the characteristics of the DIC code, including user-selectable quantities such as subset size, step size, virtual strain gage size, and other nonuser-selectable quantities such as the interpolant, subset shape function, strain calculation methods, and any filtering hidden from the user. DIC filtering is unavoidable but also 
often needed to reduce experimental noise. DIC users, therefore, need a method of understanding and representing DIC filtering, and how filtering adds bias error to DIC results and changes spatial resolution.

One possible method for quantifying spatial resolution, adopted by the DIC Challenge, is to measure the ability of a code (given a set of user-selected settings) to capture the peak displacement or strain in the sinusoidallyvarying deformation field of Sample 14. Depending on the amount of filtering of the DIC results, the amplitude of the peak will be captured well at low frequencies, but will be attenuated at higher frequencies. The cut-off frequency is the point at which attenuation begins, and the roll-off is the rate of attenuation after the cut-off frequency (using standard filter terminology). The spatial resolution is then defined as the inverse of the cut-off frequency with pixels as the units. These two quantities provide metrics for comparing the filtering effects of different DIC codes and the effects of different user-selected settings, and serve as a standard basis for understanding and presenting the spatial resolution of DIC results.

\subsection{DIC Challenge results}

\subsubsection{Sample 14}

The spatially varying displacement and strain value of Sample 14 provided in a single image the opportunity to measure both the noise level (the first columns in the left portion of the image have no displacement field) and the spatial filtering of the DIC codes with the increasing frequency displacement field in the right portion of the image. Most of the codes captured even the highest gradients in these images. Future studies from the challenge will include similar images, but with both higher displacements and higher frequencies in the displacement field to better challenge all the codes. Even with that drawback, the images provide a context for discussion on how to quantify spatial resolution in DIC measurements. (The full-field contour plots of the horizontal displacement from Codes A through K are shown in Appendix B, Figure 35). These contours give a visual sense of the displacement noise versus smoothing in the analysis. Some filtering can also be seen, particularly in Code A. Another way of visualizing the results is by taking a horizontal line-cut and averaging 50-rows as discussed previously. Those results are shown in Figure 11 and also illustrate the spatial filtering in a variety of the codes. 


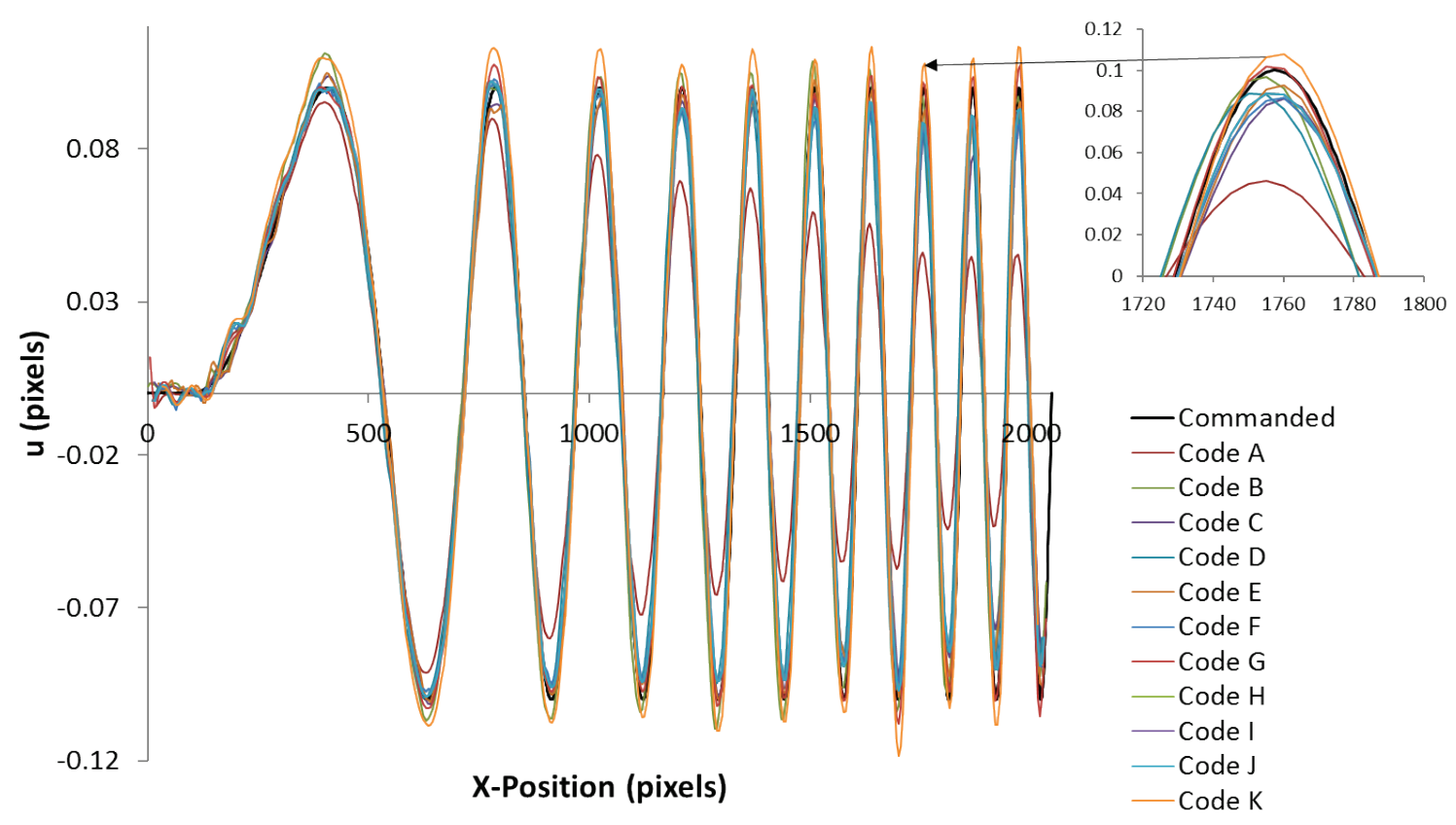

Figure 11. Line cut results showing the mean of the reported values, the commanded displacement, the bias error and the noise. The inset highlights reductions in peak amplitude caused by spatial filtering.

The full-field contour plots of the horizontal strain field are presented in Appendix B Figure 36, while the horizontal line cuts (averaged over 50 rows) are presented in Appendix B Figure 37. Strain fields always have more spatial filtering than displacement fields for two reasons. First, some number of displacement values spanning a region in space are required to compute the displacement gradients. The region of displacement data used in the strain calculations is referred to as the "virtual strain gage", and the calculated strain is the average strain in this area. Note that this same effect is seen with traditional strain gages, where the physical size of the gage averages the strain field beneath it. The virtual strain gage effect is seen regardless of the method of calculating strain, whether this is fitting a curve to a region of displacement data to get the derivative, or using neighboring points in an FE type strain calculation and then averaging, or any other process. Second, the derivative amplifies small amounts of noise in the displacement field in the resulting strain field. Therefore, a larger virtual strain gage is often required to reduce the noise in the calculated strains, which increases the spatial filtering of the strain field. 


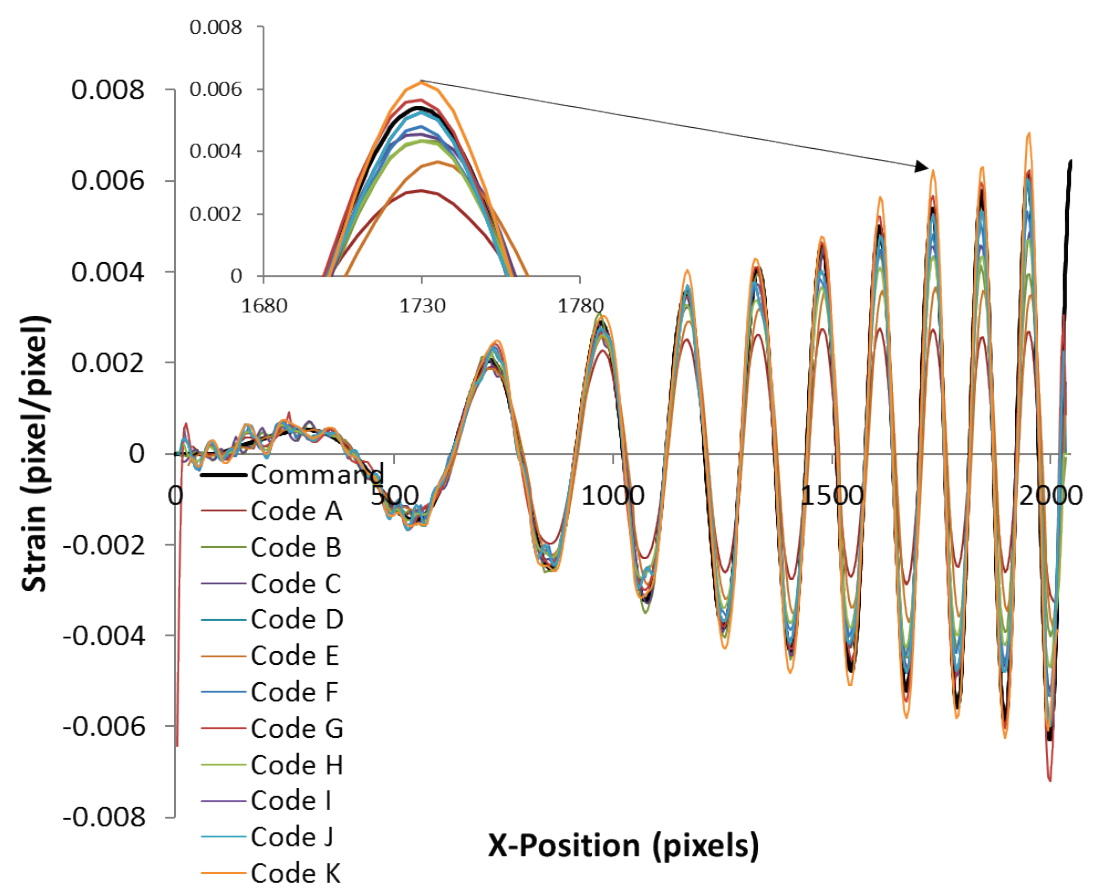

Figure 12. Line cut results showing the mean of the reported values, the commanded strain.

For Sample 14, 50 rows were averaged together and the mean and standard deviation were calculated at each reported step (that is every 5-pixels) along the columns. To compute the bias error, the measured mean displacement field was subtracted from the commanded value. The commanded value, mean measured value, bias and standard deviation curves for select codes are shown in Appendix B Figure 37. Table 4 lists the average noise for both the displacement and strain for all columns.

Table 4. Average standard deviation as a measure of overall result noise for Sample 14 L5.

\begin{tabular}{|c|c|c|}
\hline & u StDev & $\mu \varepsilon_{x x}$ StDev \\
\hline Code A & 0.005 & 172 \\
\hline Code B & 0.010 & 676 \\
\hline Code C & 0.010 & 578 \\
\hline Code D & 0.011 & 686 \\
\hline Code E & 0.015 & 256 \\
\hline Code F & 0.011 & 542 \\
\hline Code G & 0.010 & 429 \\
\hline Code H & 0.011 & 341 \\
\hline Code I & 0.012 & 638 \\
\hline Code J & 0.015 & 875 \\
\hline Code K & 0.011 & 551 \\
\hline
\end{tabular}

To better understand results, it is also useful to visualize the full-field errors and summarize them numerically. The error plotted in Figure 13 is the difference between the commanded (displacement or strain) and the measured value (Equation 3). The results in Figure 13 clearly illustrate the trade-off in filtering and bias error. Vertical noise 
is clearly seen in the full-field results and is common to all codes. The width of the banding is related to the amount of filtering. Table 5 summarizes the results using the root-mean-square error (RMSE) as defined in Equation 4. The RMSE provides an overall quality measurement by combining both the noise and the bias errors into a single metric. Also listed in the table is the absolute maximum error. This indicates the largest deviation of the measured DIC result from the commanded displacement or strain field.

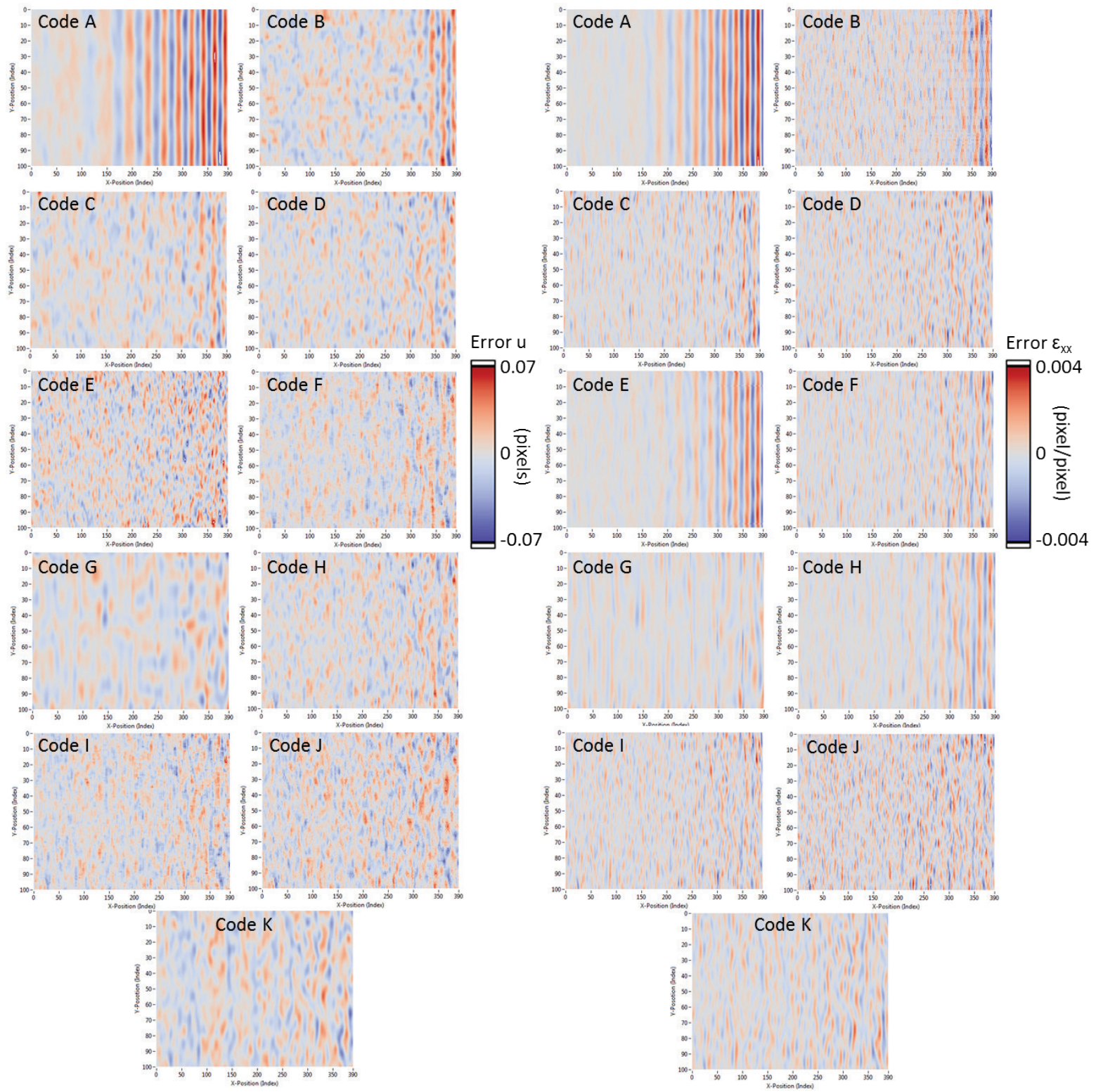

Figure 13. Full-field error plots for all the codes for both displacement (left) and strain (right). All scales are identical and the results are plotted in standard image array format to match the image deformation field. 
Table 5. Root-mean-square error (RMSE) and absolute maximum bias error.

\begin{tabular}{l|r|r|r|r} 
& RMSE u & Max Bias u & RMSE $\boldsymbol{\mu \varepsilon}_{\mathbf{x x}}$ & $\begin{array}{r}\text { Max Bias } \\
\boldsymbol{\mu} \boldsymbol{\varepsilon}_{\mathbf{x x}}\end{array}$ \\
\hline Code A & 0.022 & 0.076 & 1131 & 4129 \\
Code B & 0.014 & 0.068 & 854 & 4657 \\
Code C & 0.012 & 0.060 & 686 & 3846 \\
Code D & 0.012 & 0.058 & 754 & 3958 \\
Code E & 0.016 & 0.098 & 795 & 3405 \\
Code F & 0.013 & 0.074 & 665 & 3985 \\
Code G & 0.010 & 0.056 & 453 & 2593 \\
Code H & 0.013 & 0.070 & 601 & 3022 \\
Code I & 0.013 & 0.081 & 713 & 4415 \\
Code J & 0.015 & 0.063 & 947 & 4473 \\
Code K & 0.013 & 0.050 & 646 & 2859
\end{tabular}

\subsubsection{Determining spatial resolution from bias analysis}

In this investigation, the spatial resolution was defined as the ability of the DIC results to recreate a displacement or strain field that has a spatially varying frequency. The DIC challenge designed Sample 14 to have a 1D swept sine with constant amplitude and increasing frequency (Figure 6). The low-pass filter effect of DIC is reflected most intuitively in the roll-off of Sample 14 L5. The "roll-off" of the filter is visualized by calculating the signal attenuation as a percent of the command value as (peak or valley)/(command value). The commanded displacement peaks or valleys were set to \pm 0.1 pixels throughout the image. A LabVIEW peak detection algorithm found the peaks on both the commanded and measured displacement and strain values. The peak detection fits a quadratic function to a small region of data points to find the peak or valley location. We calculated the spatial frequency by finding the distance in pixels between neighboring peaks and valleys in the signal, both the peaks and valleys were used to have twice the number of data points across the image. The spatial period was then calculated as $2 \times$ the distance between any neighboring peaks or valleys. The percent of commanded value is plotted for the displacement as shown in Figure 14 (strain was also calculated but not shown). A $3^{\text {rd }}$ order polynomial was then fit to find the cut-off frequency. The curve was used to find the crossing point of the roll-off of either $95 \%$ or $90 \%$ of the commanded value for the displacement and strain respectively. This represents a $5 \%$ bias error in the displacement and a 10\% bias error in the strain. These values were chosen as reasonable, although arbitrary. Regardless of the levels chosen, all codes were analyzed in the same way.

Note that we have purposely chosen to treat the calculation of the spatial resolution with a "black box" approach, rather than calculating the transfer function as has been done in previous work [29,6,30]. This was done because of the multiple implementations of DIC that are analyzed in this work, including local DIC, global DIC, adaptive 


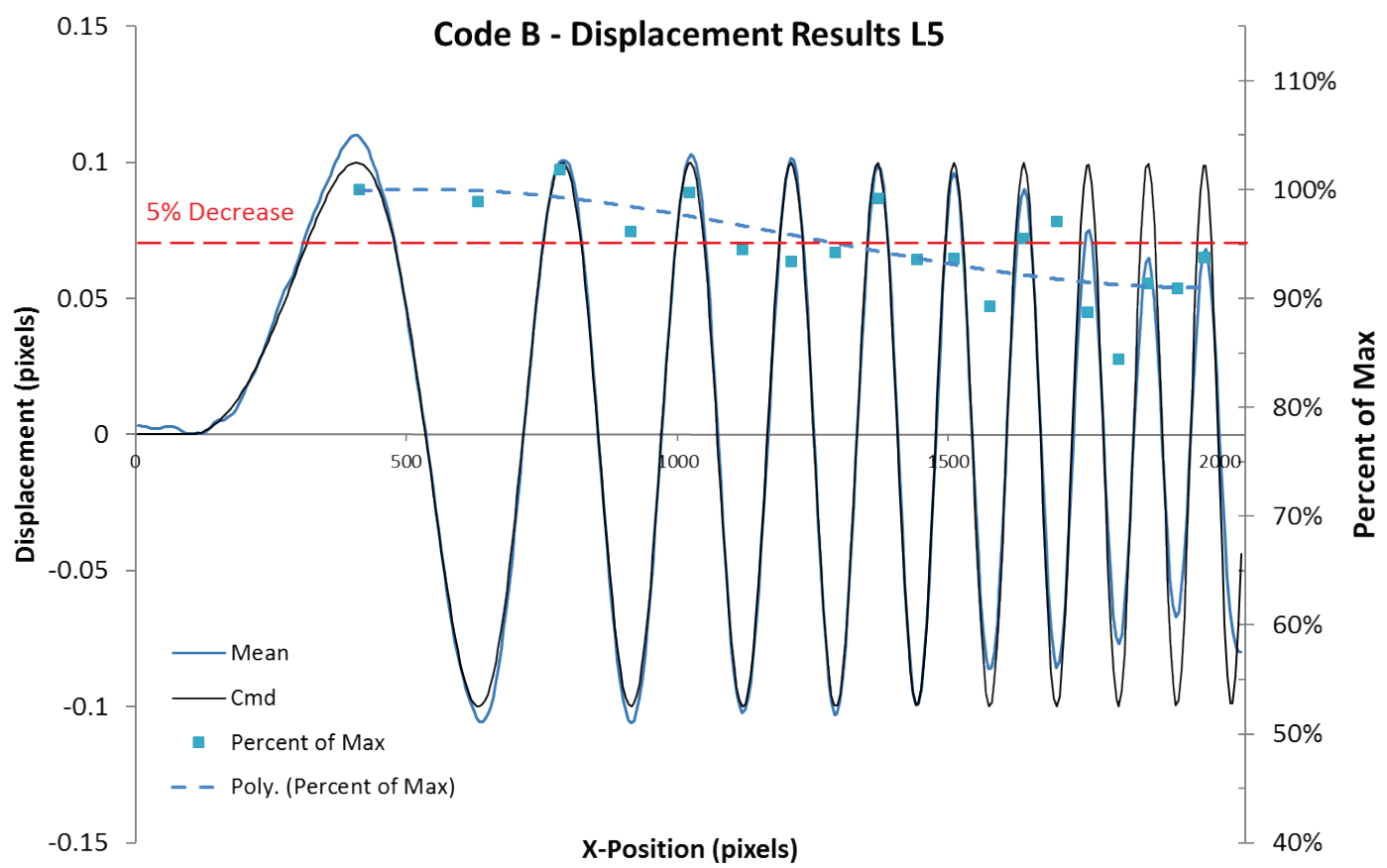

Figure 14. Displacement results for Code B for Sample 14 L5. Shows the command, mean, bias, standard deviation and percent of maximum. $3^{\text {rd }}$ order polynomial fit and $95 \%$ value shown to illustrate how the cut-off frequency was chosen.

Table 6 contains the cutoff frequencies found using the LabVIEW analysis code. The table includes the standard deviation from Table 4 to communicate an important point: All codes trade between the spatial resolution and the noise. We required the participants to choose only one data set to submit, which entails a compromise in the analysis. Different participants chose different compromises between smoothing and bias. For example, Code A has the lowest noise by a factor of at least 2, but also has the worst spatial resolution; on the other hand, Code $\mathrm{E}$ and Code $\mathrm{J}$ have the highest noise but nearly the best spatial resolution. Code $\mathrm{K}$ overestimates the displacement and strain without roll-off for these images. Most of the other codes seemed to choose about the same compromise and have nearly identical cut-off frequencies and noise levels. However, with the given requirements, it seems that Code $\mathrm{G}$ scores the best in this evaluation. 
Table 6. Cut-off frequencies (and spatial resolution) for both displacement and

strain

\begin{tabular}{|c|c|c|c|c|c|c|}
\hline & \multicolumn{3}{|c|}{ Displacement Resolution } & \multicolumn{3}{|c|}{ Strain Resolution } \\
\hline \multirow[t]{2}{*}{ Cutoff } & \multicolumn{3}{|c|}{$95 \%$} & \multicolumn{3}{|c|}{$90 \%$} \\
\hline & $\begin{array}{r}\text { Frequency } \\
(1 / \text { pixel })\end{array}$ & $\begin{array}{r}\text { Spatial Res. } \\
\text { (pixel) }\end{array}$ & $\begin{array}{r}\text { u StDev } \\
\text { (pixel) }\end{array}$ & $\begin{array}{l}\text { Frequency } \\
\text { (1/pixel) Res }\end{array}$ & $\begin{array}{l}\text { Spatial } \\
\text { s. (pixel) }\end{array}$ & $\begin{array}{r}\mu \text { exx StDev } \\
\text { (pix/pix) }\end{array}$ \\
\hline Code A & 0.0014 & 716 & 0.005 & 0.0024 & 410 & 172 \\
\hline Code B & 0.0071 & 141 & 0.010 & 0.0077 & 130 & 675 \\
\hline Code $\mathrm{C}$ & 0.0063 & 158 & 0.010 & 0.0081 & 124 & 573 \\
\hline Code D & 0.0061 & 164 & 0.011 & 0.0097 & 103 & 678 \\
\hline Code $\mathrm{E}$ & 0.0083 & 121 & 0.015 & 0.0039 & 254 & 252 \\
\hline Code F & 0.0055 & 183 & 0.011 & 0.0057 & 174 & 536 \\
\hline Code $\mathrm{G}$ & 0.0100 & 100 & 0.010 & 0.0135 & 74 & 383 \\
\hline Code $\mathbf{H}$ & 0.0055 & 182 & 0.011 & 0.0049 & 204 & 338 \\
\hline Code I & 0.0072 & 139 & 0.012 & 0.0087 & 115 & 638 \\
\hline Code $\mathrm{J}$ & 0.0096 & 104 & 0.015 & 0.0061 & 164 & 875 \\
\hline Code $\mathrm{K}$ & N/A & Over & 0.011 & N/A Ove & & 551 \\
\hline
\end{tabular}

\subsubsection{Sample 15}

Sample 15 has eight cases that could be analyzed with an increasing strain gradient on one side of a concentration and a step response to zero strain on the other side as pictured in Figure 7. We have chosen to analyze image "P200_K250.tif" for this paper as it has nicely separated peaks with a large strain value. The other images are similar with the peaks moving closer together (K50) or farther apart (K400) and with small changes in the maximum strain amplitude. For K250, the maximum strain is in the $Y$-direction ( $\left.\varepsilon_{y y}\right)$ and has a peak strain of 0.054 pixel/pixel or $54000 \mu \varepsilon$. The spatial resolution analysis conducted for Sample 14 does not apply to Sample 15; instead, we will focus on the bias errors, RMSE, and maximum acceptable bias error metric.

Consistent with Sample 14, the columns of Sample 15 were averaged across the image using a total of 188 columns to create the average (this is every other column). Sample 15 has more rows than Sample 14 because the image was wider (there was no important difference caused by this). The mean value was then subtracted from the commanded to yield the bias error as plotted in Figure 15. Table 7 contains the calculated average standard deviation for the line cut and the maximum absolute value of the bias error. 


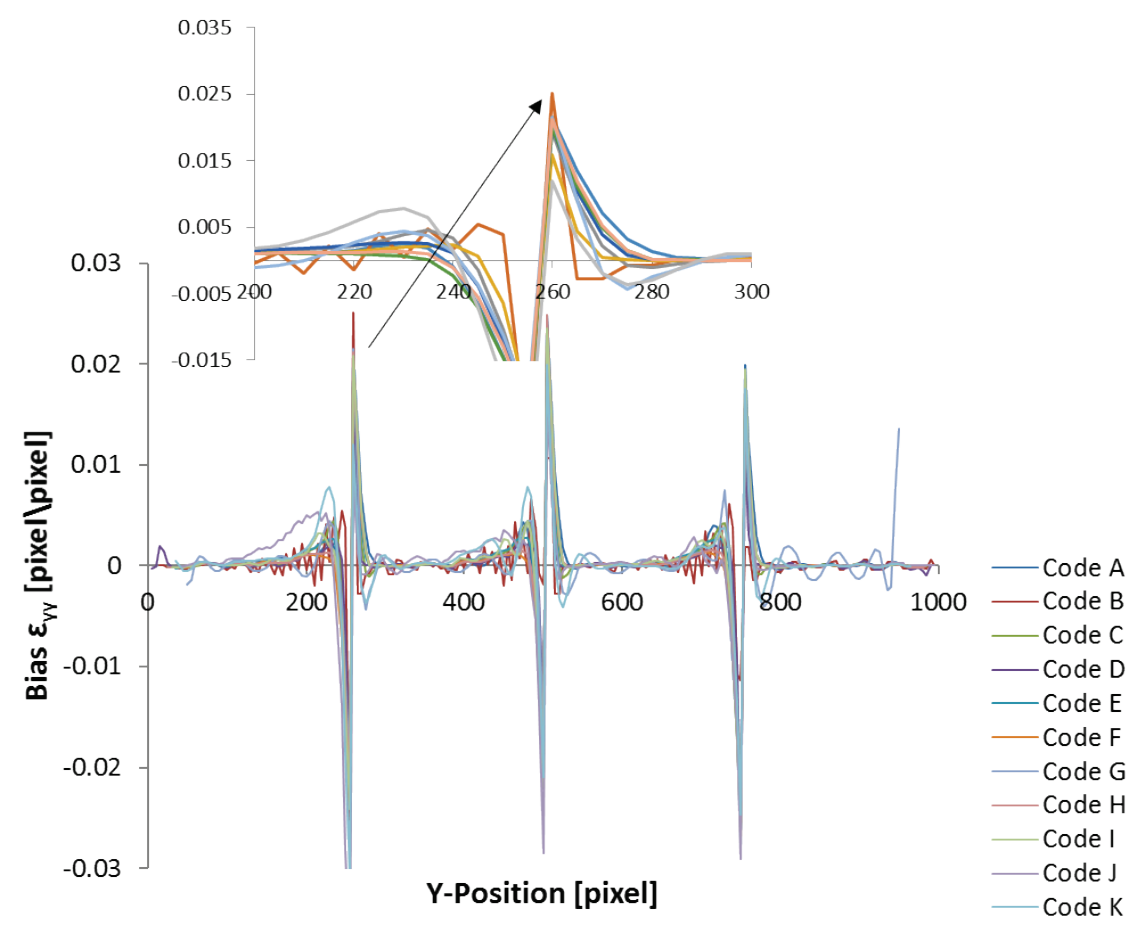

Figure 15. Bias error for Sample $15 \mathrm{~K} 250$ for all codes. This is the mean of 188 columns subtracted from the command value. Note the large error at the discontinuity.

Table 7. Average standard deviation and absolute maximum bias error from the line cuts. Data corresponds to the line cuts in Figure 15.

\begin{tabular}{r|cr|rr} 
& \multicolumn{2}{|c|}{$\mathbf{v}$} & \multicolumn{2}{|c}{$\varepsilon_{\mathrm{yy}}$} \\
& $\begin{array}{l}\text { Average } \\
\text { St Dev }\end{array}$ & Max Bias & \multicolumn{2}{|l}{ Average } \\
& St Dev & Max Bias \\
\hline Code A & 0.0111 & 0.273 & 0.0008 & 0.026 \\
Code B & 0.0256 & 0.089 & 0.0041 & 0.029 \\
Code C & 0.0101 & 0.185 & 0.0008 & 0.026 \\
Code D & 0.0179 & 0.125 & 0.0008 & 0.026 \\
Code E & 0.0110 & 0.191 & 0.0008 & 0.031 \\
Code F & 0.0159 & 0.146 & 0.0006 & 0.027 \\
Code G & 0.0096 & 0.159 & 0.0008 & 0.028 \\
Code H & 0.0163 & 0.155 & 0.0006 & 0.025 \\
Code I & 0.0196 & 0.224 & 0.0015 & 0.026 \\
Code J & 0.0143 & 0.363 & 0.0008 & 0.038 \\
Code K & 0.0122 & 0.191 & 0.0009 & 0.031
\end{tabular}

The full-field difference plots are shown in Figure 16 for both the displacement and strain. The range of the color bar is set to be $\pm 5 \%$ of the full-scale commanded displacements and $\pm 10 \%$ of the full-scale commanded strains. While there are some differences in how the codes handle the noise and the discontinuity, the basic features were similar for all codes. Namely, some displacement and strain data is outside the predetermined range near the discontinuity, with larger errors on either side of the discontinuity (except for Code B, which had random noise distribution spatially). Table 8 presents the RSME, the maximum bias, and the percent of reported points that were within the predetermined range. This table again illustrates the tradeoff between filtering and bias errors. 
Code B for example does a better job of capturing the peak displacement (low maximum bias) but has more noise throughout the image. Other codes, such as Code G, have much lower noise throughout the field, but still miss some region of the peak strain and displacement.

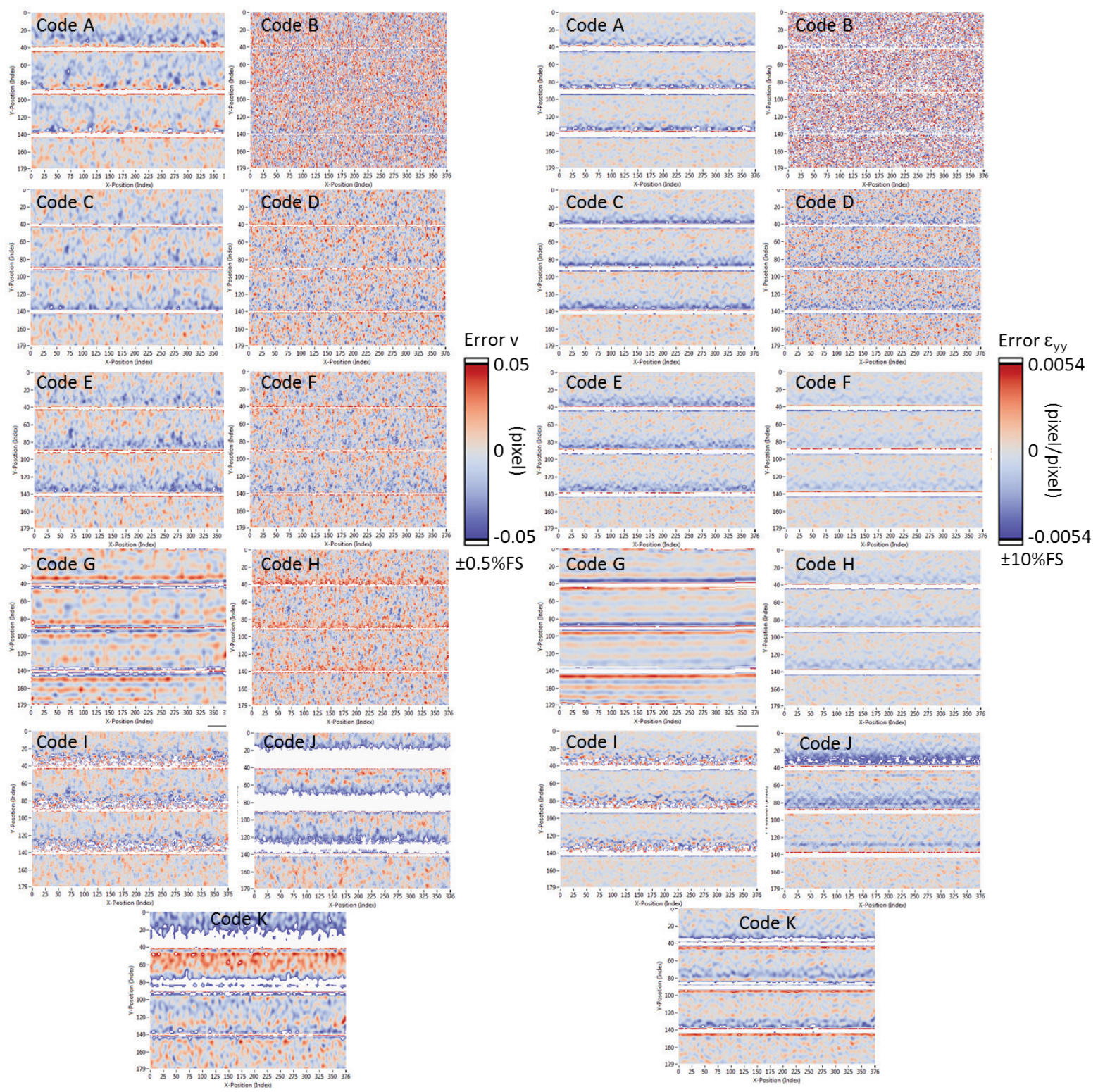

Figure 16. Full-field error plots for the displacement (left) and strain (right). Difference between commanded and DIC results. The scales represent $\pm 0.5 \%$ of full-scale for displacement and $\pm \mathbf{1 0} \%$ full-scale for strain. These correspond to the limits in Table 8 . White regions in the data field are points either above or below the plot ranges, rather than missing data. 
Table 8. RMSE error for both displacement and strain for the full-field data shown in Figure 16.

\begin{tabular}{|c|c|c|c|c|c|c|}
\hline & RMSE v & $\begin{array}{r}\text { ax Bias } \\
v\end{array}$ & $\begin{array}{r}\% \text { within } \\
\pm 0.05 \\
\text { pixels }\end{array}$ & MSE $\varepsilon$ & $\begin{array}{r}\text { Max Bias } \\
\varepsilon_{\mathrm{yy}}\end{array}$ & $\begin{array}{r}\% \text { within } \\
10 \% \text { max } \\
\text { Strain }\end{array}$ \\
\hline Code A & 0.045 & 0.273 & $92 \%$ & 0.0047 & 0.030 & $92 \%$ \\
\hline Code B & 0.028 & 0.089 & $93 \%$ & 0.0055 & 0.043 & $76 \%$ \\
\hline Code C & 0.028 & 0.185 & $95 \%$ & 0.0045 & 0.028 & $93 \%$ \\
\hline Code D & 0.023 & 0.125 & $97 \%$ & 0.0042 & 0.032 & $94 \%$ \\
\hline Code $\mathrm{E}$ & 0.030 & 0.191 & $95 \%$ & 0.0047 & 0.030 & $92 \%$ \\
\hline Code $\mathrm{F}$ & 0.024 & 0.146 & $97 \%$ & 0.0049 & 0.028 & $91 \%$ \\
\hline Code G & 0.024 & 0.159 & $96 \%$ & 0.0047 & 0.030 & $92 \%$ \\
\hline Code $\mathrm{H}$ & 0.027 & 0.155 & $96 \%$ & 0.0048 & 0.027 & $92 \%$ \\
\hline Code I & 0.042 & 0.224 & $89 \%$ & 0.0055 & 0.040 & $88 \%$ \\
\hline Code J & 0.100 & 0.363 & $67 \%$ & 0.0058 & 0.041 & $91 \%$ \\
\hline Code K & 0.047 & 0.191 & $81 \%$ & 0.0049 & 0.033 & $90 \%$ \\
\hline
\end{tabular}

\section{Conclusions}

It is human nature to compete - and therefore a "score" seems necessary. However, scoring is not the primary purpose of the DIC Challenge, and the challenge board did not rank the codes from 1 to $N$. We seek to provide a methodology and defined metrics to assess DIC codes that will be used in future publications. The development of thought on how to quantify spatial resolution is a good example of this progress. However, we think presenting the performance relative to several different metrics will allow a view of how well a code is performing for a given application. The following list of goals is more important for the challenge board than a score.

1. A verified and validated set of images (and corresponding displacement fields) for people to use in publications and code development. The errors already discovered regarding Eulerian versus Lagrangian coordinate systems are an illustration of the importance of this!

2. An understanding of the compromise in DIC code between filtering and noise, also known as the regularization length.

3. Motivation for improvements in DIC codes where necessary, particularly for university codes.

4. Defined metrics that have a broad community consensus on how to quantify a "good" DIC result.

5. A formation of a consistent vocabulary (e.g., "spatial resolution"). See a recent paper by Grédiac that discusses this in detail [30].

6. A methodology for weighing pros and cons of different approaches: Global versus Local, etc. 
The preliminary sample DIC challenge image sets and analysis were an important first step in helping the DIC community to better understand what influences the quality of their DIC results. All the image sets provide a basis for evaluating the DIC software with different sets providing different challenges to the DIC algorithm; noise robustness, lack of contrast, interpolation errors and so forth. These images can be used for software verification. Verification is defined as testing the code to see if it is functioning correctly. The images cannot be used for experimental validation. That is, these images will not quantify the expected uncertainty in an experiment. Many of the error sources are missing in the synthetic images, which only include image noise, and therefore are not relevant for determining experimental error bounds. Rather, the challenge images would be the "best case scenario" in an experiment for a given speckle pattern and image noise. Remember the images analyzed for the challenge (Sample 14 and Sample 15) are synthetic images! The errors determined here may be only a small portion of the error budget for many experiments. Anything less than $1 / 100^{\text {th }}$ of a pixel of error is likely not going to be seen in an actual physical experiment. On the other hand, the experimental image sets do include all the errors that occur, but either do not have known results or are simplistic in their displacement fields (e.g., rigid body displacement).

Scoring of the codes in this paper is incomplete. Some have said that for a typical analysis, the experimentalist would optimize the DIC software settings with an often better defined goal in using the results. This can often be done with a virtual strain gage size study varying the parameters to investigate the filtering effect, balanced against the need to contain noise. In fact, this is how one should typically conduct their post processing. However, it is the balance between the noise and bias that is difficult to determine. In defense of the approach the board has taken, let us note that in most analysis, you probably want both great spatial resolution and minimized noise! We do not think that the task set for the analysis of these images is substantively different than for a typical experiment: You have a set of images and want to get the best results possible. Even if you are going to use this to calculate material properties as an integrated step, the balance of noise and bias will be an important and maybe poorly understood compromise.

We cannot emphasize enough the goal of this exercise was not to rank the codes - but provide a forum for understanding how DIC is working. We think that it is generally true that given different solution parameters, each of the 10 codes could be brought to be even closer to each other in performance. That is, the quality of the results for 2D-DIC depend more on the selection of the DIC software analysis settings than it does on the implementation of the code (at least for those participating in this challenge). Therefore, training is an important component in adopting DIC as a primary measurement technique in the lab. It is easy to get answers from DIC, it 
is harder to always get good answers from DIC. It should also be emphasized that for many experiments the quality of the results is driven far more by issues with the experiment - than issues with the DIC code. Out-ofplane errors [31] and heat waves [32] are just two examples that will most often overwhelm the errors within the DIC implementation.

It is hoped that all future publications would use DIC Challenge image sets when making claims of improvement in the quality of the results. Without a common set of images, there is no meaningful method of comparison. Note that both the images used and the software required to analyze the images are freely available on the SEM website. As reviewers, we should be insisting on the use of these images in order to be able to draw meaningful conclusions from papers on 2D-DIC accuracy.

\section{Future Goals of the DIC Challenge}

After the publication of this paper, a final round of images will be provided that are blind. These blind images will be based on Sample 14 and Sample 15 (see Table 1), but with deformation fields unknown to the participants. These images will be provided to all who desire to participate, with the results to be published in a subsequent paper. The advantage of blind image sets is that any possibility of impropriety is removed from the analysis portion by the participants. Additionally, standard image sets for stereo-DIC are currently being designed, and the 2DDIC Challenge will be extended to stereo-DIC. Preliminary image sets, including both calibration images and deformed images, are at the Challenge website. There will be both synthetic images created using the Balcaen simulator and completely experimental image sets. The experimental image sets will be used for round-robin code comparison and for measurements against secondary diagnostics. The Challenge Board invites your comments and ideas.

\section{Acknowledgements}

The 2D-DIC Challenge is dedicated to Dr. Laurent Robert. An active and important board member in the early years of the project, who passed away in 2016. He has been sorely missed in the experimental mechanics community. The Challenge would like to thank, Francois Hild, Stephane Roux and Bernd Wieneke for pointing out the Lagrange/Euler discrepancy and suggesting solutions to that problem.

Sandia is a multiprogram laboratory operated by Sandia Corporation, a Lockheed Martin Company, for the United States Department of Energy's National Nuclear Security Administration under contract No. DE-AC0494AL85000. 


\section{References}

1. Chu T, Ranson W, Sutton M (1985) Applications of digital-image-correlation techniques to experimental mechanics. Exp Mech 25 (3):232-244. doi:10.1007/bf02325092

2. Bruck H, McNeill S, Sutton M, Peters W (1989) Digital image correlation using Newton-Raphson method of partial differential correction. Exp Mech 29 (3):261-267. doi:10.1007/bf02321405

3. Luo PF, Chao YJ, Sutton MA, Peters WH, III (1993) Accurate measurement of three-dimensional deformations in deformable and rigid bodies using computer vision. Exp Mech 33 (2):123-132.

doi:10.1007/BF02322488

4. Helm JD, McNeill SR, Sutton MA (1996) Improved three-dimensional image correlation for surface displacement measurement. Opt Eng 35 (7):1911-1920. doi:10.1117/1.600624

5. Bay BK, Smith TS, Fyhrie DP, Saad M (1999) Digital volume correlation: Three-dimensional strain mapping using X-ray tomography. Exp Mech 39 (3):217-226. doi:Doi 10.1007/Bf02323555

6. Sutton DA, Orteu JJ, Schreier HW (2009) Image Correlation for Shape, Motion and Deformation

Measurements. Springer, New York, NY

7. Reu P (2012) Hidden Components of DIC: Calibration and Shape Function - Part 1. Experimental Techniques 36 (2):3-5. doi:10.1111/j.1747-1567.2012.00821.x

8. Reu P (2012) Introduction to Digital Image Correlation: Best Practices and Applications. Experimental Techniques 36 (1):3-4. doi:10.1111/j.1747-1567.2011.00798.x

9. Hild F, Roux S (2012) Comparison of Local and Global Approaches to Digital Image Correlation. Exp Mech 52 (9):1503-1519. doi:10.1007/s11340-012-9603-7

10. Stanislas M, Okamoto K, Kahler C (2003) Main results of the First International PIV Challenge. Meas Sci Technol 14 (10):R63-R89. doi:Pii S0957-0233(03)54192-3

11. Stanislas M, Okamoto K, Kähler C, Westerweel J, Scarano F (2008) Main results of the third international PIV Challenge. Exp Fluids 45 (1):27-71. doi:10.1007/s00348-008-0462-z

12. Stanislas M, Okamoto K, Kähler CJ, Westerweel J (2005) Main results of the Second International PIV Challenge. Exp Fluids 39 (2):170-191. doi:10.1007/s00348-005-0951-2

13. BIPM I, IFCC I, IUPAC I, ISO O (2008) The international vocabulary of metrology - basic and general concepts and associated terms (VIM), 3rd edn. JCGM 200: 2012. JCGM (Joint Committee for Guides in Metrology)

14. Oberkampf WL, Barone MF (2006) Measures of agreement between computation and experiment: validation metrics. J Comput Phys 217 (1):5-36. doi:10.1016/j.jcp.2006.03.037

15. Reu P (2011) Experimental and Numerical Methods for Exact Subpixel Shifting. Exp Mech 51 (4):443-452. doi:10.1007/s11340-010-9417-4

16. Orteu J-J, Garcia D, Robert L, Bugarin F A speckle texture image generator. In: Pierre S, Christine C (eds), 2006. vol 1. SPIE, p $63410 \mathrm{H}$

17. Lehmann TM, Gonner C, Spitzer K (1999) Survey: Interpolation methods in medical image processing. Ieee T Med Imaging 18 (11):1049-1075. doi:Doi 10.1109/42.816070

18. Baldi A, Bertolino F (2015) Experimental analysis of the errors due to polynomial interpolation in digital image correlation. Strain 51 (3):248-263

19. Bornert M, Doumalin P, Dupré J-C, Poilane C, Robert L, Toussaint E, Wattrisse B (2017) Shortcut in DIC error assessment induced by image interpolation used for subpixel shifting. Opt Laser Eng 91:124-133

20. Grédiac M, Sur F (2014) 50th Anniversary Article: Effect of Sensor Noise on the Resolution and Spatial Resolution of Displacement and Strain Maps Estimated with the Grid Method. Strain 50 (1):1-27. doi:Doi 10.1111/Str.12070

21. Wang Y, Lava P, Debruyne D (2015) Using super-resolution images to improve the measurement accuracy of DIC.

22. Reu P (2015) All about Speckles: Contrast. Experimental Techniques 39 (1):1-2. doi:10.1111/ext.12126

23. Wang YQ, Sutton MA, Bruck HA, Schreier HW (2009) Quantitative Error Assessment in Pattern Matching: Effects of Intensity Pattern Noise, Interpolation, Strain and Image Contrast on Motion Measurements. Strain 45 (2):160-178. doi:DOI 10.1111/j.1475-1305.2008.00592.x

24. Cox RW, Raoqiong T (1999) Two- and three-dimensional image rotation using the FFT. Image Processing, IEEE Transactions on 8 (9):1297-1299

25. Fazzini M, Mistou S, Dalverny O, Robert L (2010) Study of image characteristics on digital image correlation error assessment. Opt Laser Eng 48 (3):335-339

26. Passieux J-C, Bugarin F, David C, Périé J-N, Robert L (2015) Multiscale Displacement Field Measurement Using Digital Image Correlation: Application to the Identification of Elastic Properties. Exp Mech 55 (1):121137. doi:10.1007/s11340-014-9872-4 
27. Boyce BL, Reu PL, Robino CV (2006) The constitutive behavior of laser welds in 304L stainless steel determined by digital image correlation. Metallurgical and Materials Transactions A (Physical Metallurgy and Materials Science) 37A (8):2481-24922492

28. Bornert M, Bremand F, Doumalin P, Dupre JC, Fazzini M, Grediac M, Hild F, Mistou S, Molimard J, Orteu JJ, Robert L, Surrel Y, Vacher P, Wattrisse B (2009) Assessment of Digital Image Correlation Measurement Errors: Methodology and Results. Exp Mech 49 (3):353-370. doi:10.1007/s11340-008-9204-7

29. Schreier HW, Sutton MA (2002) Systematic errors in digital image correlation due to undermatched subset shape functions. Exp Mech 42 (3):303-310

30. Grédiac M, Blaysat B, Sur F (2017) A critical comparison of some metrological parameters characterizing local digital image correlation and grid method. Exp Mech

31. Sutton MA, Yan JH, Tiwari V, Schreier HW, Orteu JJ (2008) The effect of out-of-plane motion on 2D and 3D digital image correlation measurements. Opt Laser Eng 46 (10):746-757.

doi:10.1016/j.optlaseng.2008.05.005

32. Reu P, Toussaint E, Jones EM, Bruck HA, Iadicola MA, Balcaen R, Turner DZ, Siebert T, Lava P, Simonsen M (In Review) DIC Challenge: Developing Images and Guidelines for Evaluating Accuracy and Resolution of 2D Analyses. Exp Mech 


\section{Appendix A}

In this section, example analyses and some comments are provided for Image Sets 1-13 and 16-17. These DIC results were performed by the lead author (not by the DIC Challenge participants), using a single, commerciallyavailable DIC software. These results are intended to provide examples of analysis techniques and typical results expected from these images for others who intend to use the image sets to test their own DIC code.

\section{A.1 Sample 1 and Sample 5 - Rigid-body-motion with contrast variation.}

Sample 1 and 5 are both rigid body shifted images with varying contrast and noise (1.5 counts) in an 8-bit image. The images are shifted in both $x$ - and $y$-directions simultaneously in shifts of 0.05 pixels/step (Sample 1 - see Figure 17) and 0.1 pixels/step (Sample 5 - see Figure 18). Figure 17 shows the histogram for Sample 1 for the first and last image. Sample 1 provides a varying contrast throughout the image series. Most DIC codes cannot correlate throughout the entire series unless a ZNSSD algorithm is used because the contrast shift is different for each image. It is important for DIC codes to compensate for contrast shifts because in most DIC experiments, particularly for stereo-DIC, there will be changes in the contrast during the experiment.

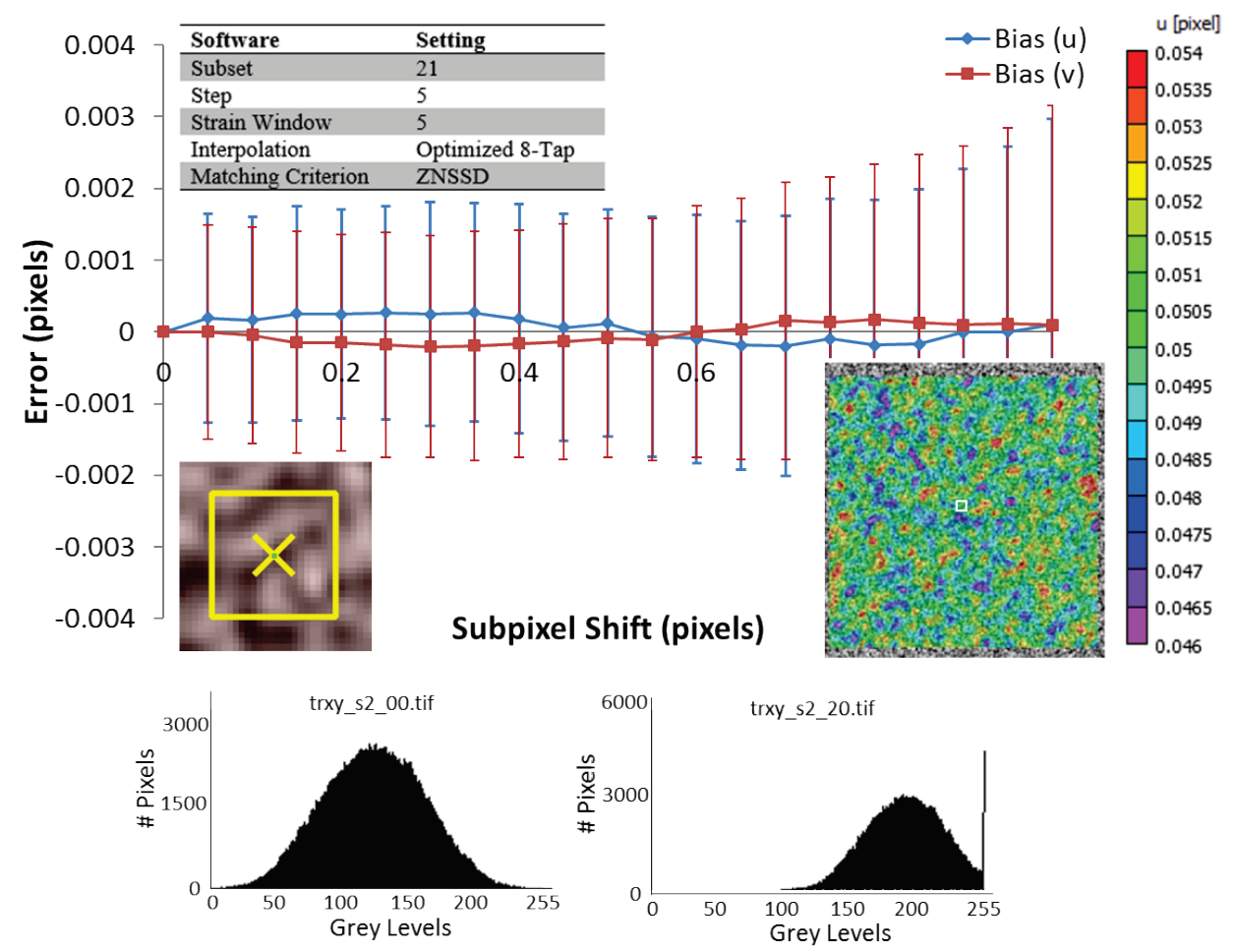

Figure 17. Bias and variance results for Sample 1. Inset in the figure are the DIC analysis parameters and the full-field results for file "trxy_s2_01.tif" with the subset size pictured on the speckle pattern. Bottom of figure is the histogram for the first and last image of the series as labeled. Error bars are based on $+/$ - one standard deviation. 


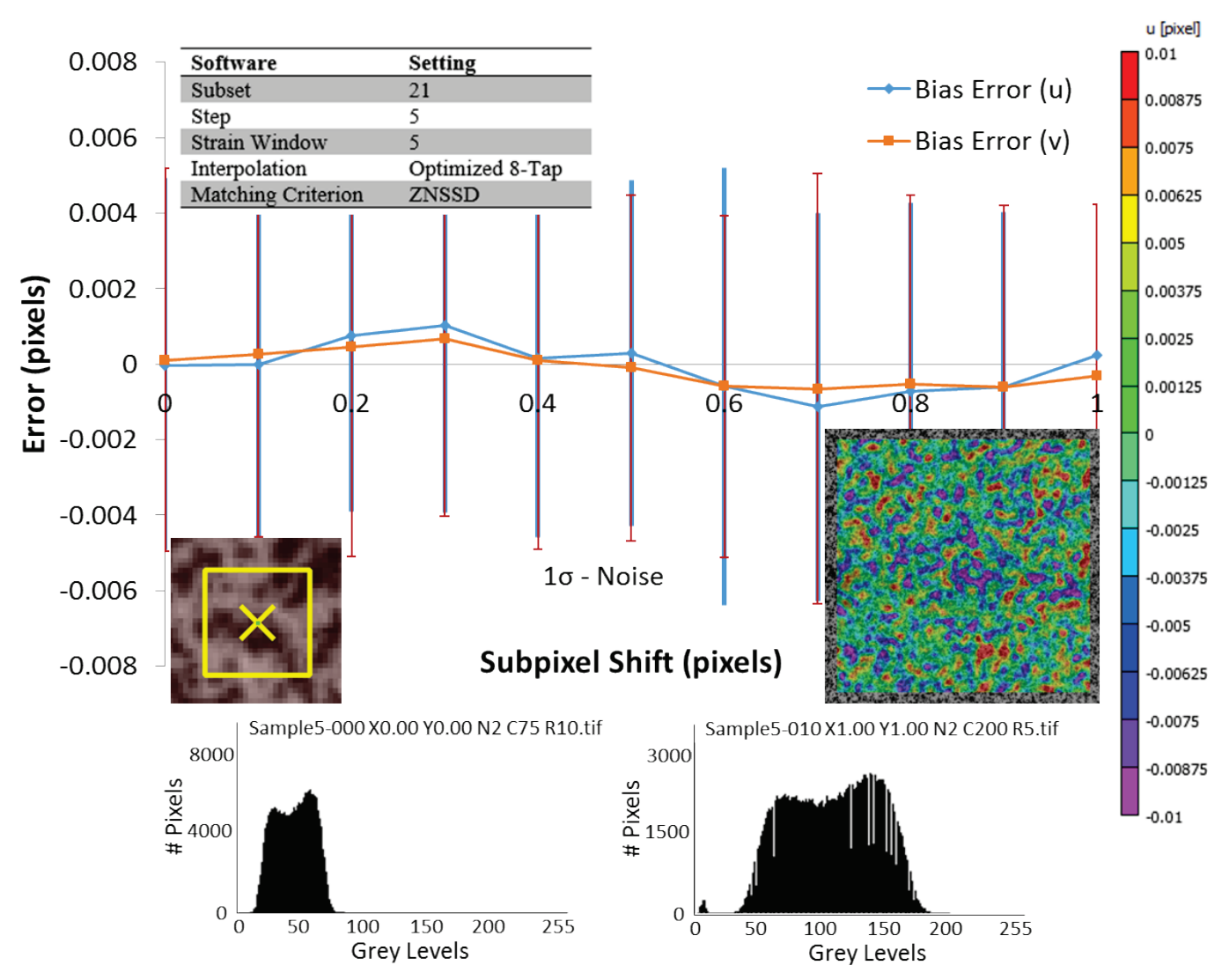

Figure 18. Bias and variance results for Sample 5. Inset color plot is for "Sample5000 X0.00 Y0.00 N2 C75 R10.tif". Bottom of figure is the histogram for the first and last image of the series as labeled. Error bars are based on +/- one standard deviation.

\section{A.2 Sample 2 and Sample 4 - Rigid-body-motion with high image noise}

Sample 2 and Sample 4 were created to have rigid-body shifts with poor contrast and very high noise. Results for Sample 2 are shown in Figure 19 and for Sample 4 are shown in Figure 20. 


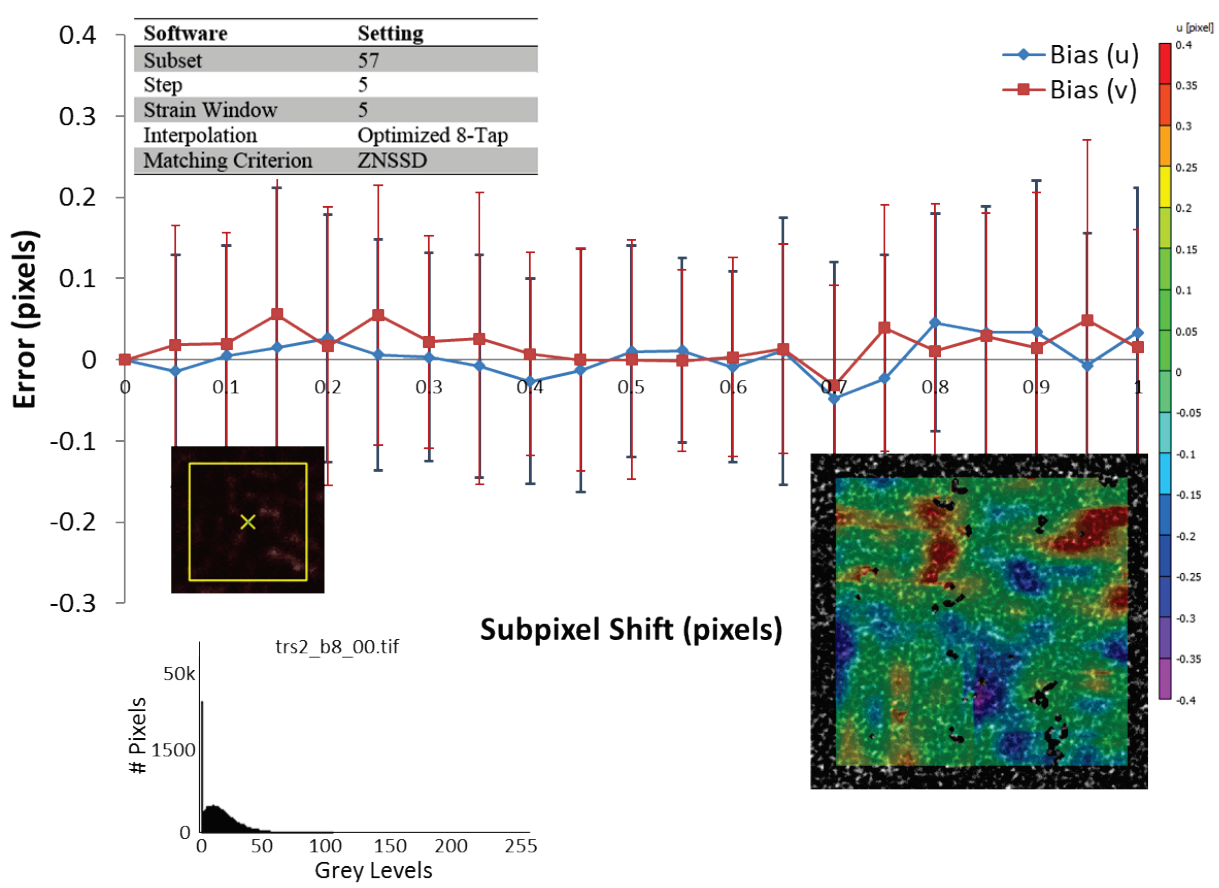

Figure 19. Sample 2 results. Insets show the DIC settings and the results from "trs2_b8_01.tif". The DIC results are plotted over a reduced range of contrast to make the speckles visible. The subset is shown over the speckle pattern, note that the speckles are barely visible.

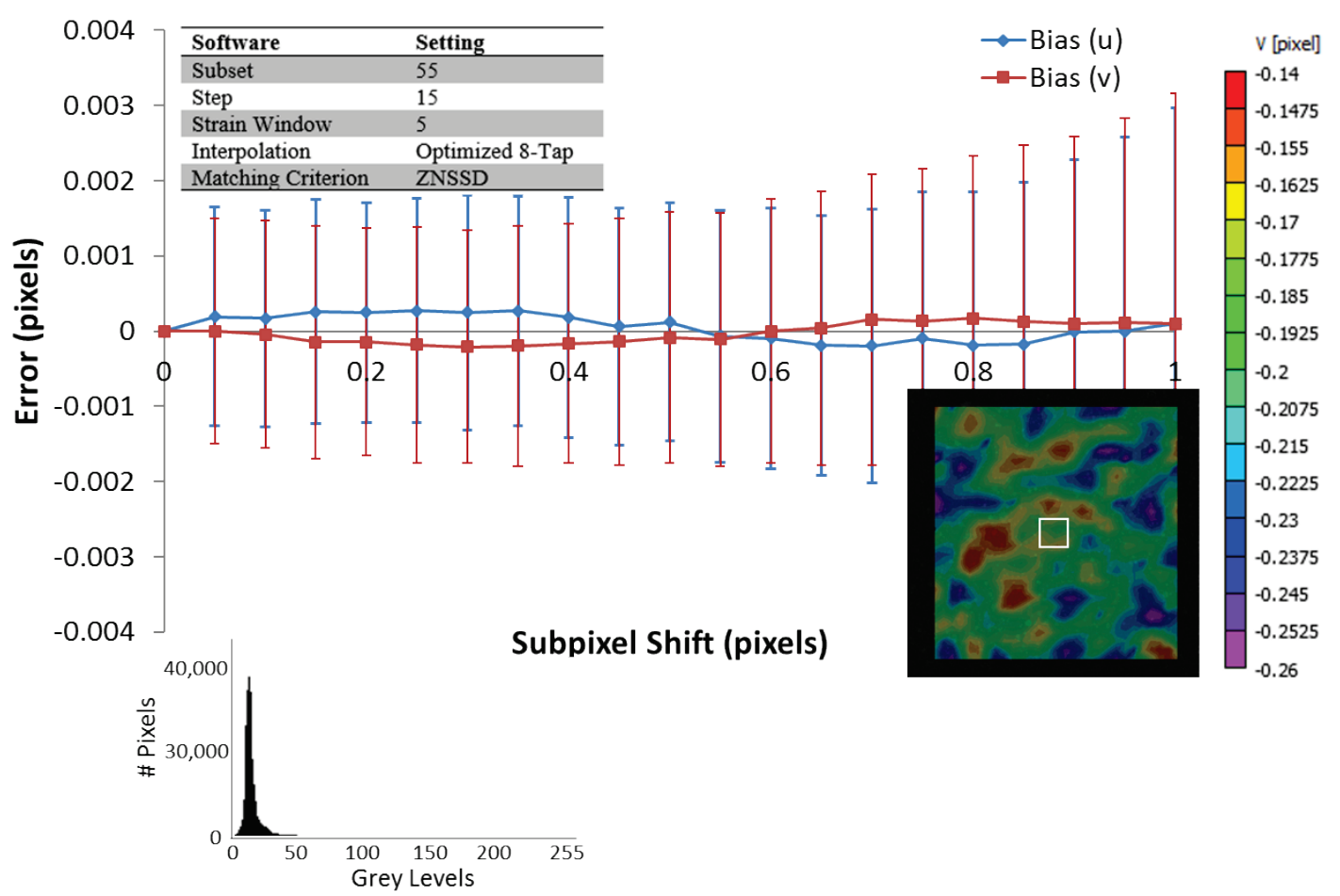

Figure 20. Sample 4 results for image "Sample4-002 X0.2 Y0.2 N8 C50 R0.tif". Inset in the figure are the image (which appears nearly black due to the poor contrast) with the large subset (white square) required to get correlation to work as indicated in the DIC settings. Bottom of figure is the histogram for the first image of the series as labeled. Error bars are based on $+/$ - one standard deviation. 


\section{A.3 Sample 3 and Sample 3b - FFT Rigid-body-motion and step shift}

Sample 3 is an FFT shifted image with a typical noise level and can be used to look at the variance and bias errors (shown in Figure 21).

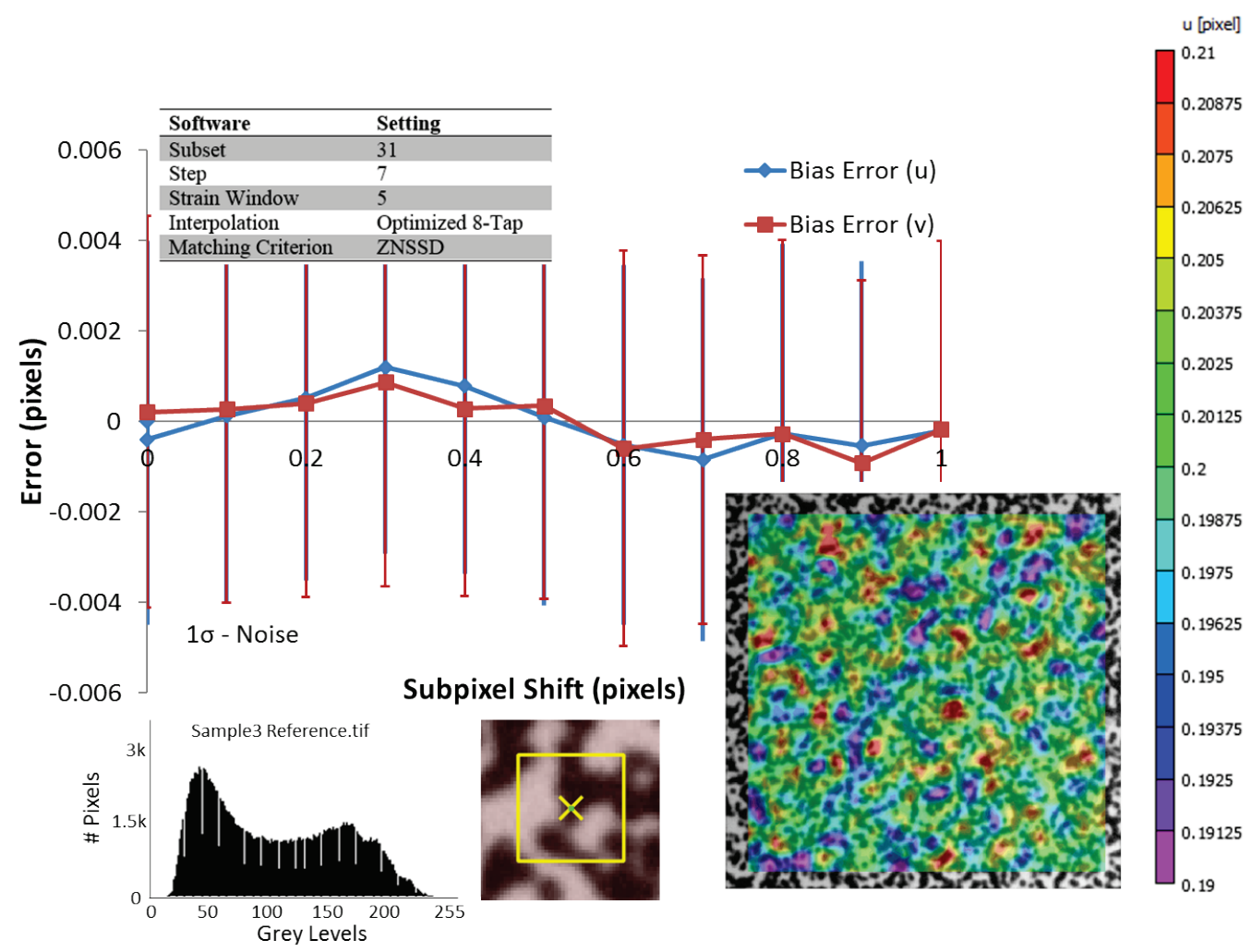

Figure 21. Bias and variance results for Sample 3. Inset shows the DIC settings and results from "Sample3-002 X0.20 Y0.20 N2 CO R0.tif". Subset is shown over the speckle pattern. Bottom of figure is the histogram for the first image of the series as labeled. Error bars are based on $+/-$ one standard deviation.

Sample $3 \mathrm{~b}$ shifts half of the image by $0.05,0.1,0.2,0.25$ and 0.5 pixels. Results are shown in Figure 22 for all 5 shifts using the DIC settings shown in the inset. A study on the effect of changing the step size and the subset size are shown in Figure 23. It can be seen that larger subsets and steps create a larger roll-off at the discontinuity. 

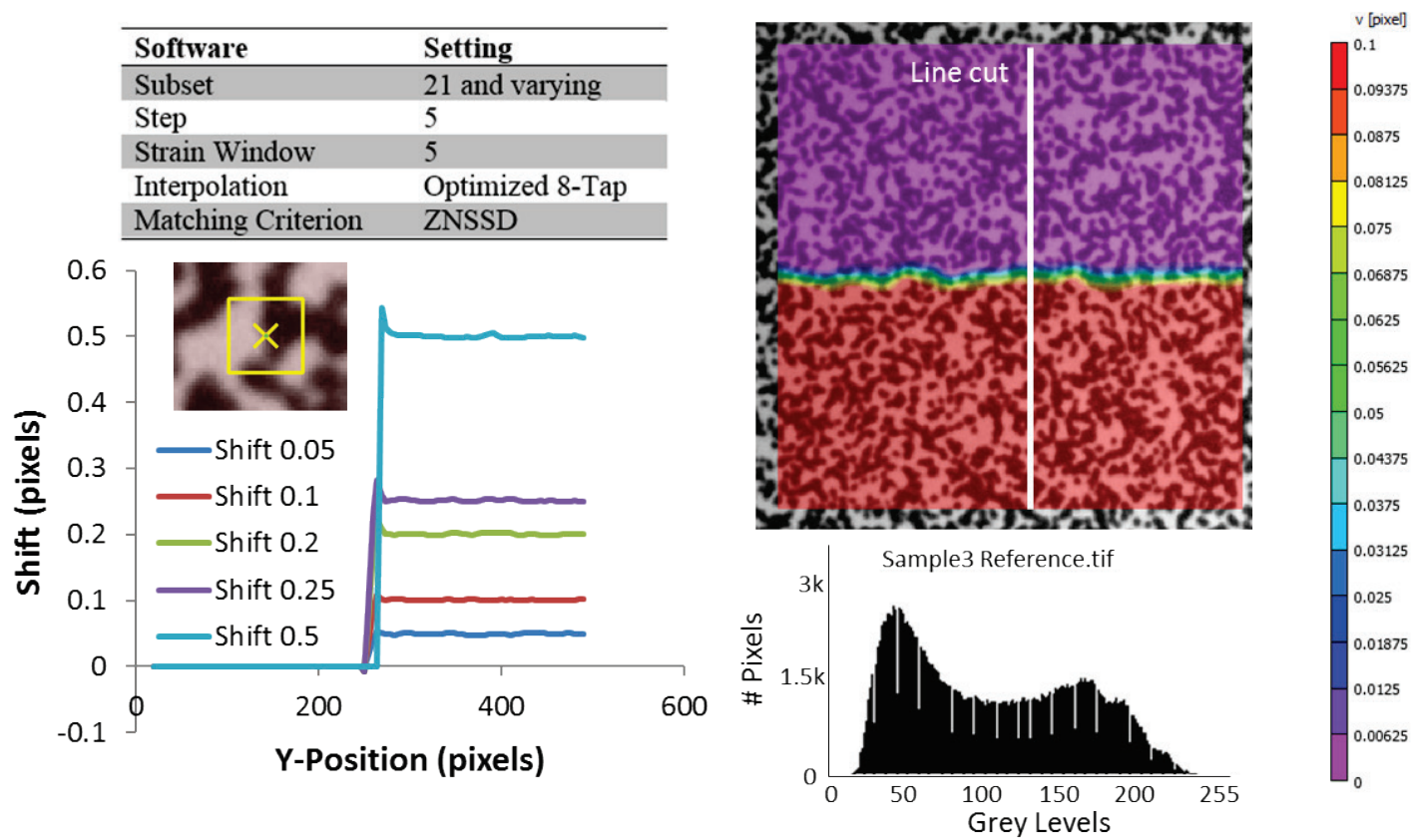

Figure 22. Results from all Sample $3 b$ line cuts. Full-field results shown for "Sample3 Half01.tif" with the line cut shown. Histogram of the reference image and DIC settings also shown.

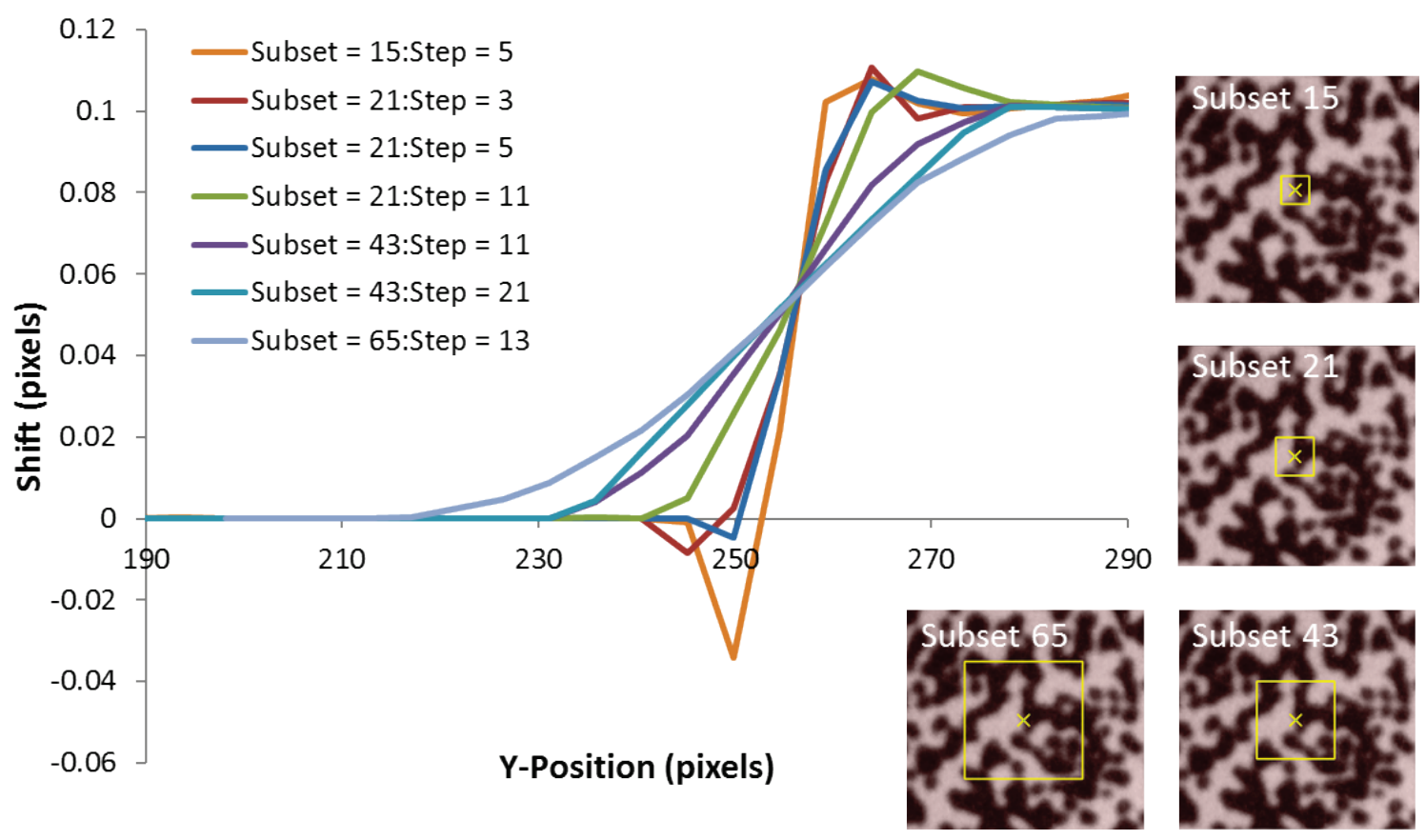

Figure 23. Close up of the shift profiles near the discontinuity for a subset and step size study using the "Sample3 Half01.tif" image and the line cut (in Figure 23). Subset from smallest to largest are shown inset on the speckle pattern. 


\section{A.4 Sample 6 and Sample 7 - Pseudo-experimental image shifting}

Sample 6 and Sample 7 were created using the pseudo-experimental approach. Note that the errors are much larger for Sample 7 than for the similar synthetically generated Samples 2 and 4. As the pseudo-experimental results mimic more realistically the errors that are witnessed in a typical DIC experiment, it prompts the question: What is being missed in the synthetic image creation process?

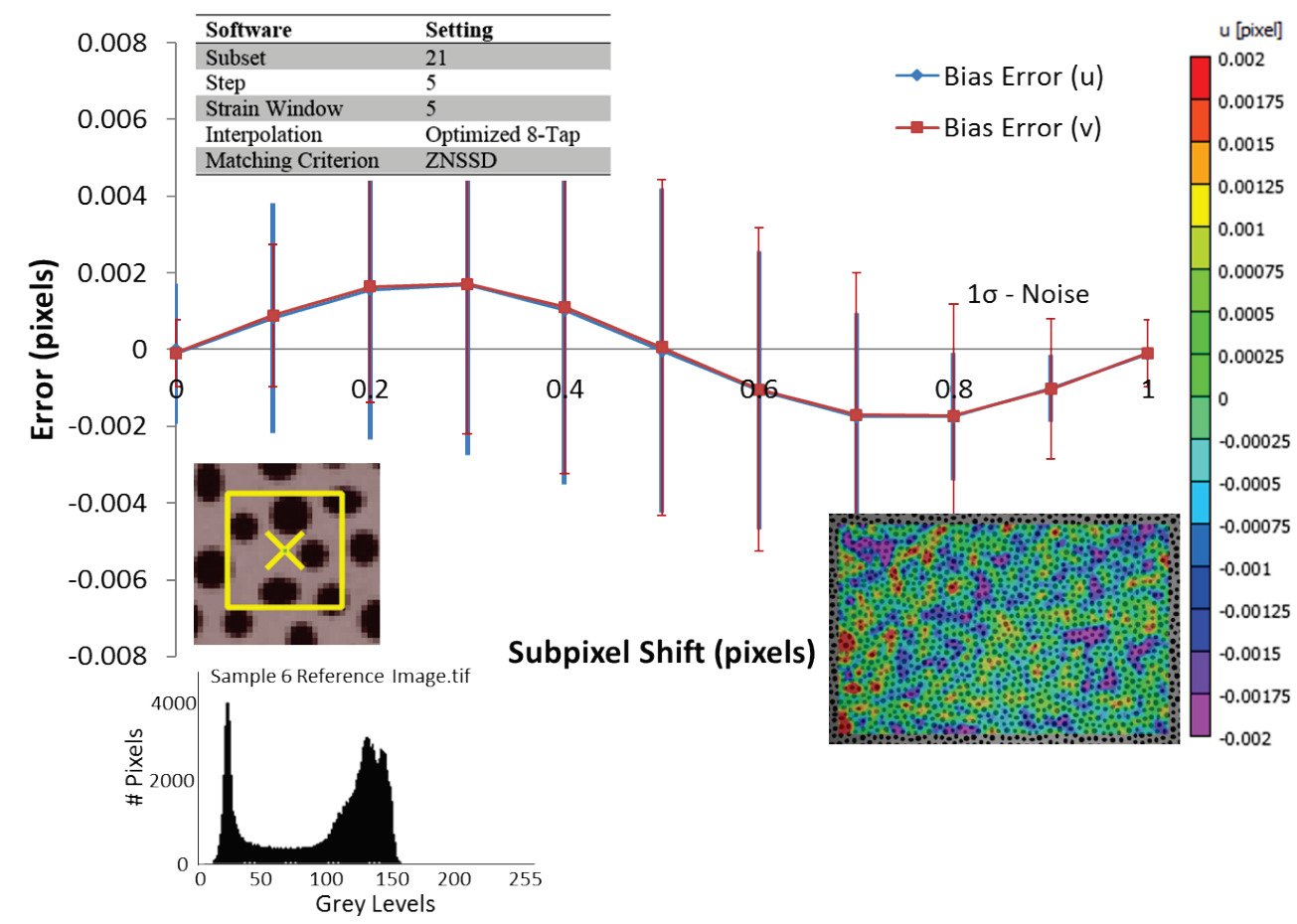

Figure 24. Sample 6 results for low-noise pseudo-experimentally shifted images. Inset DIC results are for "Sample6-Pro Dot G0_Y00 X00.tif". Inset shows speckle pattern with subset overlay. Histogram also shown. Error bars are based on $+/-$ one standard deviation. 


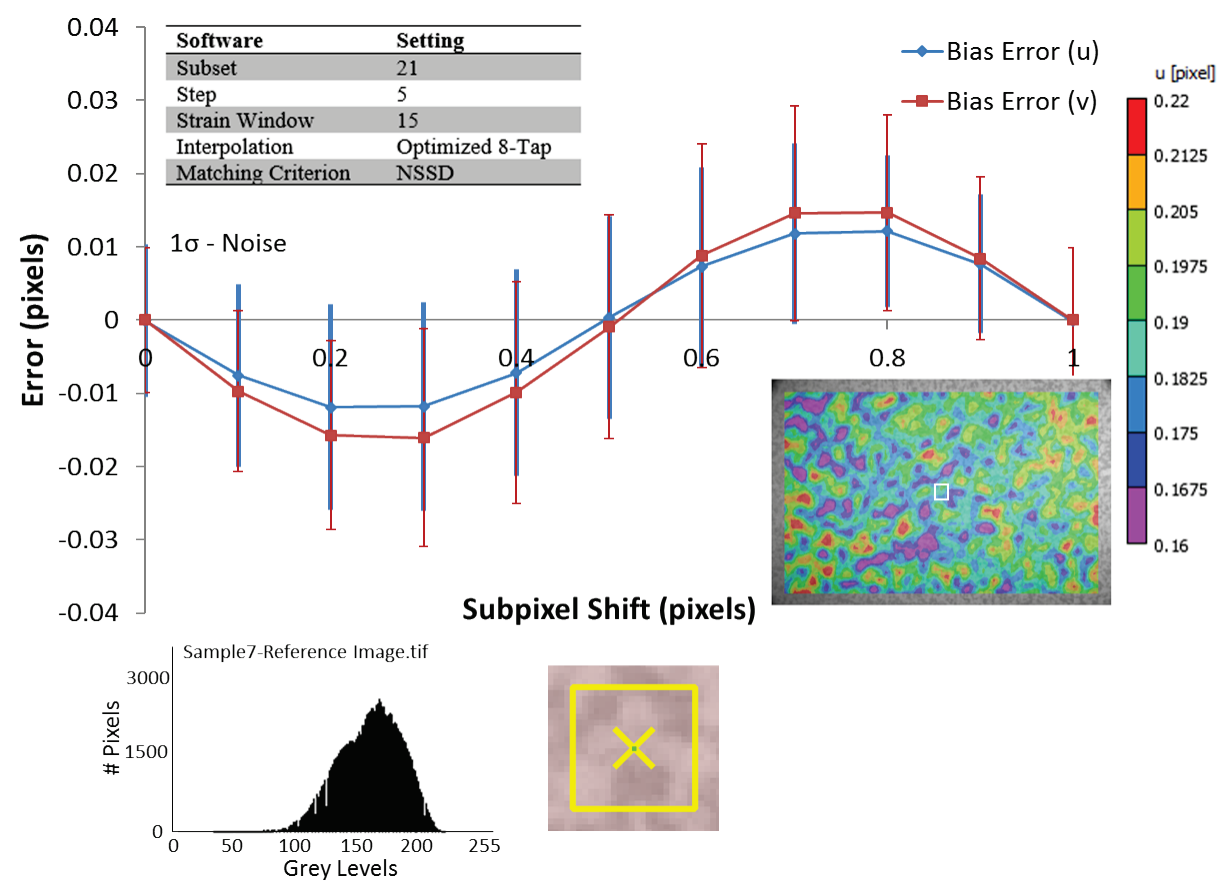

Figure 25. Sample 7 results for image "Sample7-LCG25-Y02-X02.tif" are shown in the inset with the subsite size illustrated. DIC parameters are inset as well. Bottom of figure is the histogram for the first image of the series as labeled. Error bars are based on $+/$ - one standard deviation.

\section{A.5 Sample 8 and Sample 9 - Image rotation}

For Sample 8 and 9 the rotation mean and standard deviation were calculated at each subset step using the following equation:

$$
\theta=\tan ^{-1} \frac{\sqrt{u^{2}+v^{2}}}{\sqrt{(x-256)^{2}+(y-256)^{2}}} \cdot \frac{180}{\pi}
$$

The results are plotted in angle error, defined as the difference between the known angle and the measured angle: $\left(\theta_{\text {DIC }}-\theta_{\text {synthetic }}\right)$. Figure 26 are the results for Sample 8 and Figure 27 for Sample 9. The results are nearly identical, with the results from Sample 9 being noisier as indicated by the larger error bars representing one standard deviation $(1 \sigma)$ of the angle error. Sample 9 has a slightly larger angle noise due most likely to the sub-optimal painted speckle pattern verses the nearly ideal speckle pattern created by TexGen. 


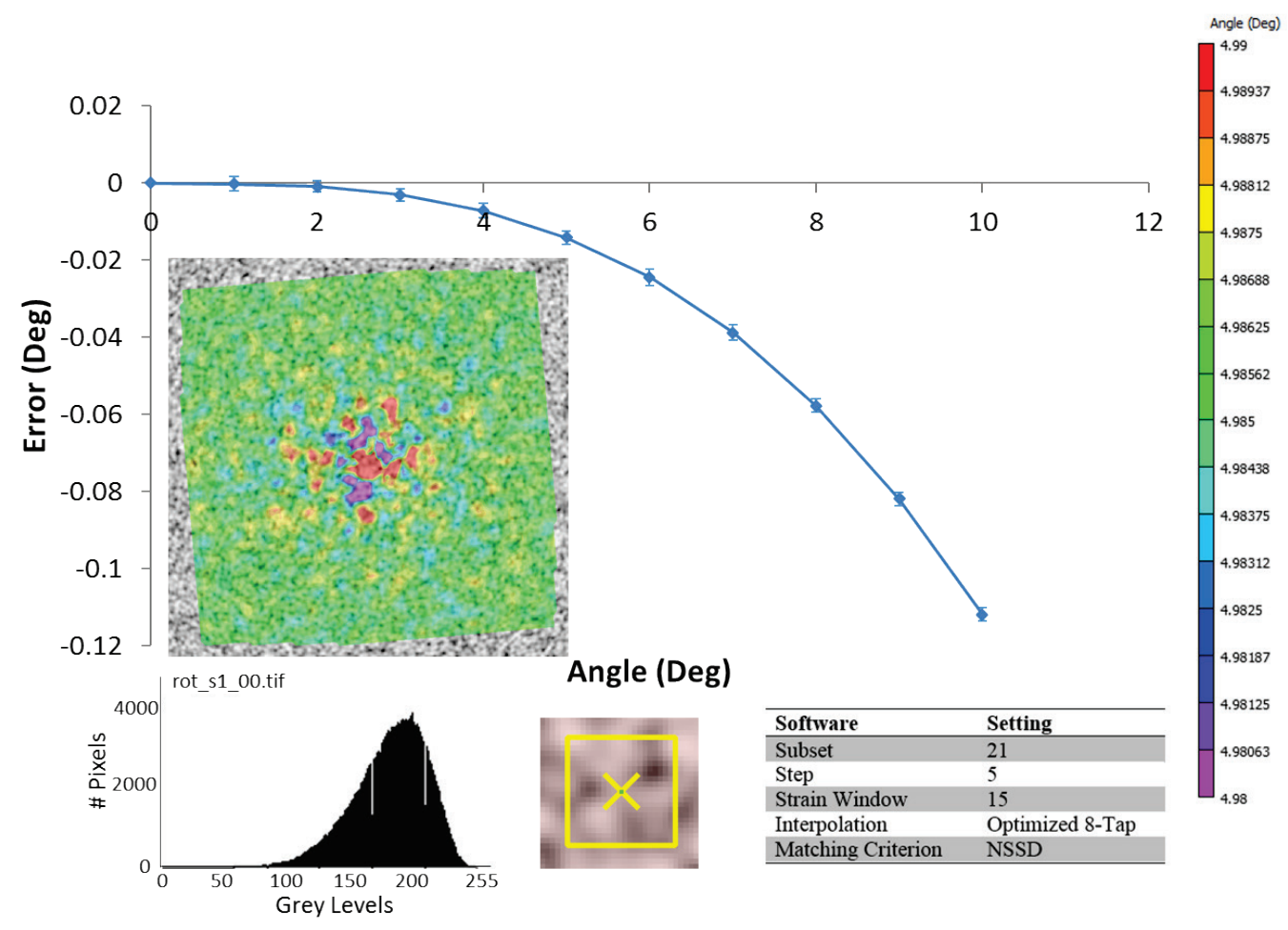

Figure 26. Sample 8 TexGen rotation results. Inset DIC results for "rot_s1_05-tif". Subset overlay on speckle pattern, histogram and DIC settings also displayed. Error bars are based on $+/-$ one standard deviation.

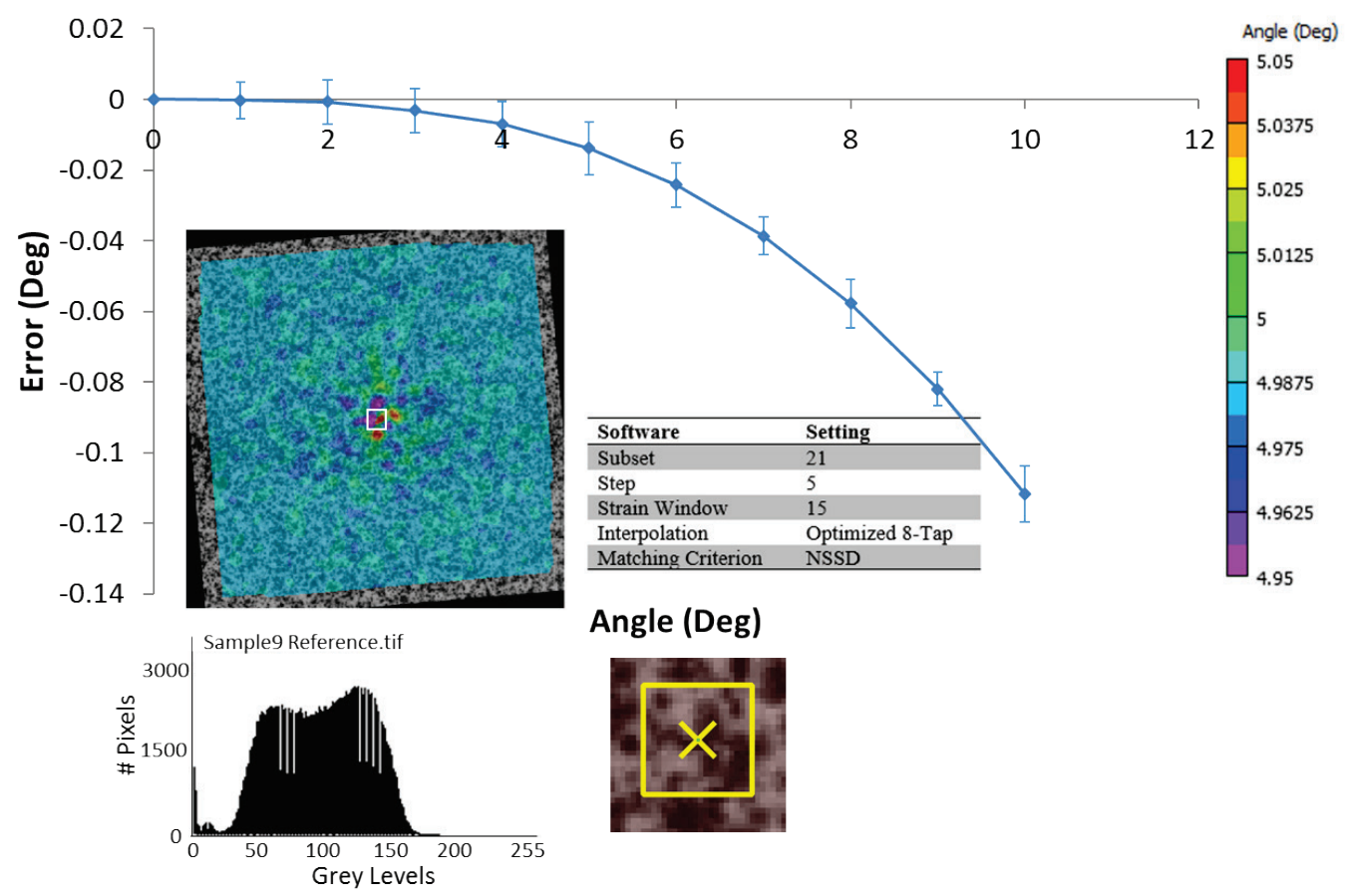

Figure 27. Sample 9 FFT rigid body rotation results for image "Sample9-05.tif". Inset in the figure are the full-field measured results for the $5^{\circ}$ case, with the DIC software settings listed. Bottom of figure is the histogram for the first image of the series as labeled. Error bars are based on $+/$ - one standard deviation. 


\section{A.6 Samples 10, 11, 11b - Spatially varying strain field}

Sample 10 and Sample 11 used a spatially varying function from Fazzini and Laurent [25].

Sample $11 \mathrm{~b}$ was created with a triangle shaped displacement field. This creates a step in the strain field that can be used to investigate the roll-off of the DIC filtering. Typical results with the DIC settings can be found in Figure 28.

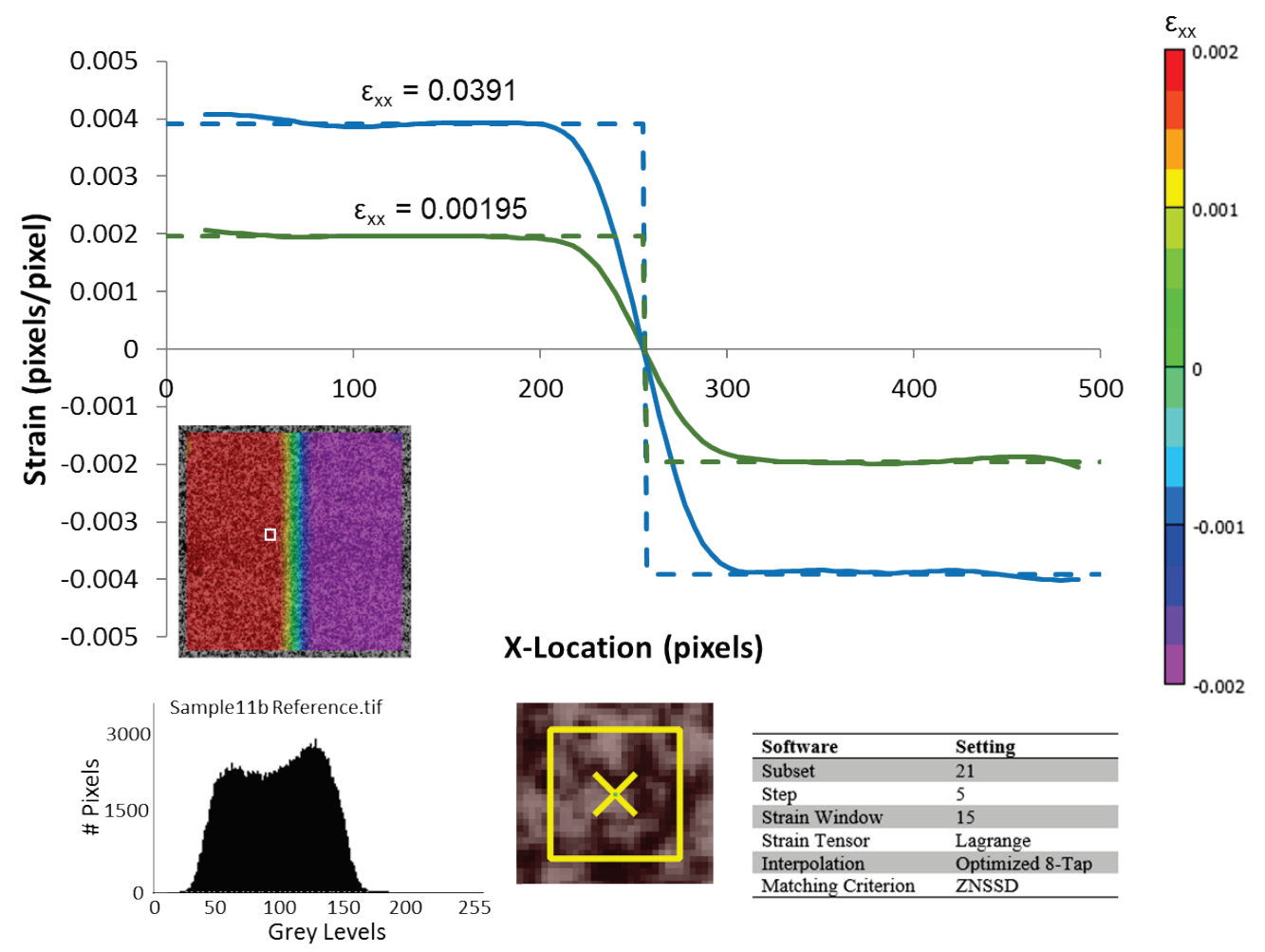

Figure 28. Sample 11b results for the triangle wave function created using the FFT. Inset is the full-field strain results for image "Sample 11b Tri-0.5.tif", which corresponds to a strain of $\mathbf{0 . 0 0 1 9 5}$. Software settings for the analysis are inset as well. Commanded results are dotted lines, DIC reconstructed solid lines of the same color. Bottom of figure is the histogram for the first image of the series as labeled.

\section{A.7 Samples 12 and 13 - Experimental image series}

Figure 29 shows a cross-sectional data cut through the center of the specimen for Sample 12 with the principal strain plotted. The subset, step and strain window (filter) were varied to demonstrate the importance of these parameters on the spatial resolution conducting a typical virtual strain gage size study. More details on the specimen and material may be found in [26]. 
A full-field result from Sample 13 is shown in Figure 30. Incremental correlation was used with the DIC settings shown in the figure. Strain in the $y$-direction is plotted. More details on the experiment can be found in [27].

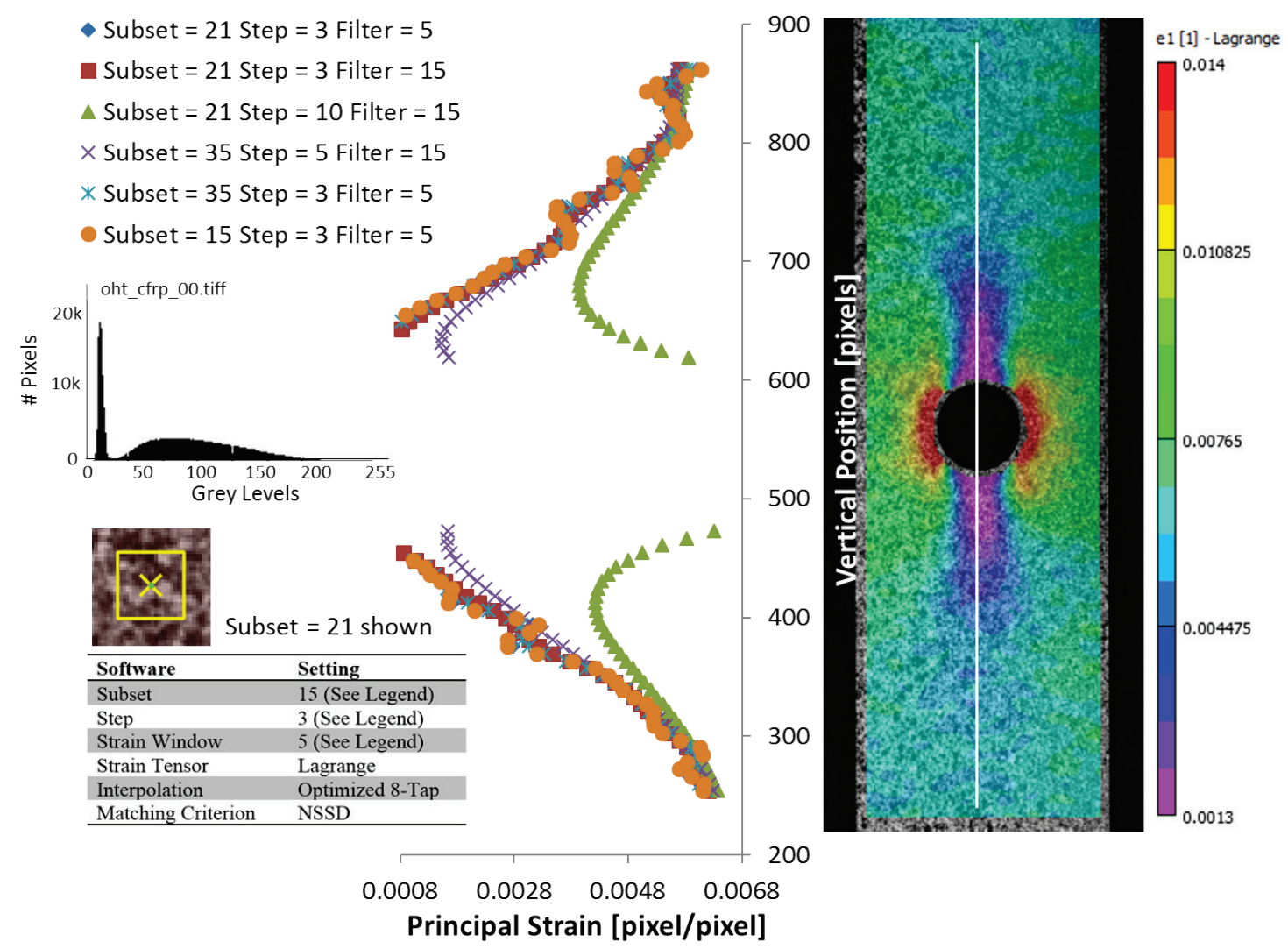

Figure 29. Sample 12 results for image “oht_cfrp_11.tiff”. Inset full-field data is for subset $=15$, step $=3$, strain window $=5$. Line cut plots shown taken vertically through the center of the specimen. Also shown the histogram for the first image of the series as labeled. 

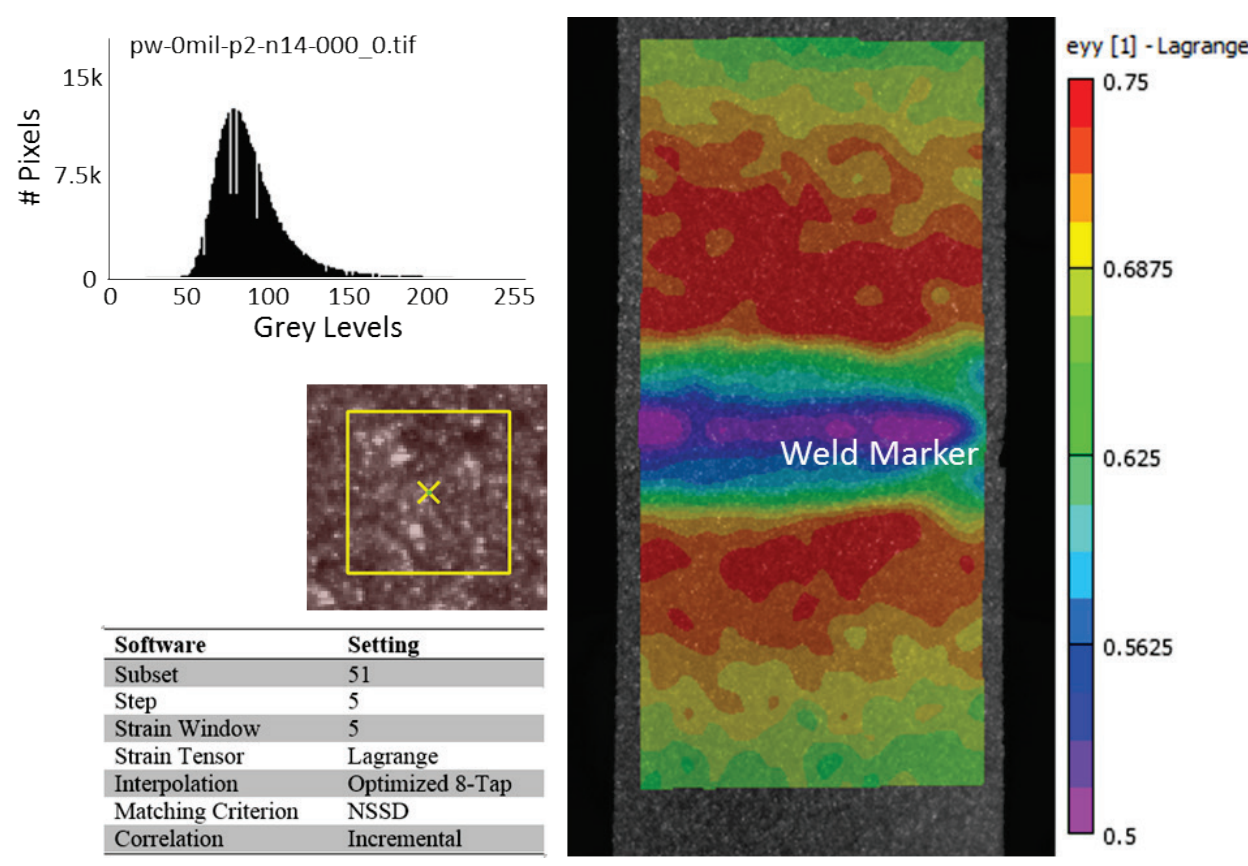

Figure 30. Sample 13 results for image "pw-0mil-p2-n14-660_0.tif”. Strain in the vertical direction is shown using the inset DIC software settings. Also shown the histogram for the first image of the series as labeled.

\section{A.8 Sample 16 - Experimental in-plane translation}

Sample 16 was a rigid-body experimental translation data set. The experimental description and setup are detailed in the text and shown in Figure 4. Displacement results for two steps, one early in the translation and one towards the end are shown in Figure 31 and were analyzed using 3 common DIC interpolants (Figure 35). Also reported in the figure are the stage position error and standard deviation. The interpolation bias error can be clearly seen in the DIC results (Figure 35). The final complication is the lens distortions which began to grow as the sample was translated. Even with small lens distortions ( \pm 0.03 pixels at 1-pixel translation), they caused the displacement error to be incorrectly reported (see Figure 34 image 00050). However, we assume that the mean value reported is still correct. Therefore, the error bars we report in Figure 32 are the $+/$ - one standard deviation noise from the first 0.01-pixel step before lens distortions become noticeable and biased the noise results.

Note the bias errors are extremely small. The ability to experimentally measure the bias errors (for a good interpolant) is extremely difficult. To our knowledge, there is only one other experiment reported in the literature where the bias errors can be clearly seen in an experiment using a translation stage [18]. To achieve this required control of the lab environment, a high-precision stage, and the image noise had to be reduced via binning and averaging the images. This is a cautionary tale regarding the relative importance of the bias errors versus the other experimental errors. For comparison we show a more typical experimental result in Figure 33 where the identical 
setup was used with PointGrey cameras. We conducted the same shifting experiment but without averaging and binning of the images. Here the variance error can be seen to dominate and the interpolation bias error is no longer visible in the results.

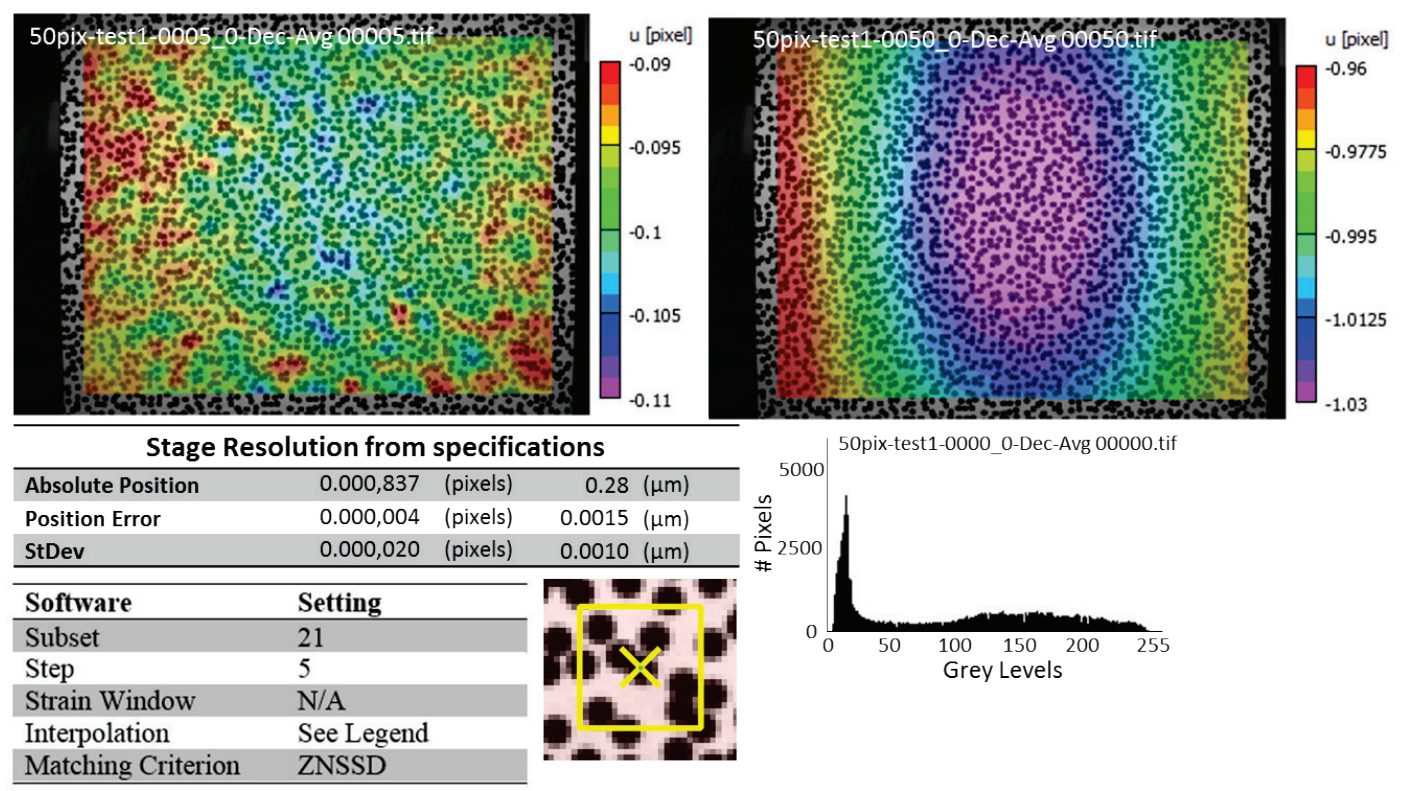

Figure 31. Experimental setup and stage specifications. DIC full-field results show the lens distortions at the last image.

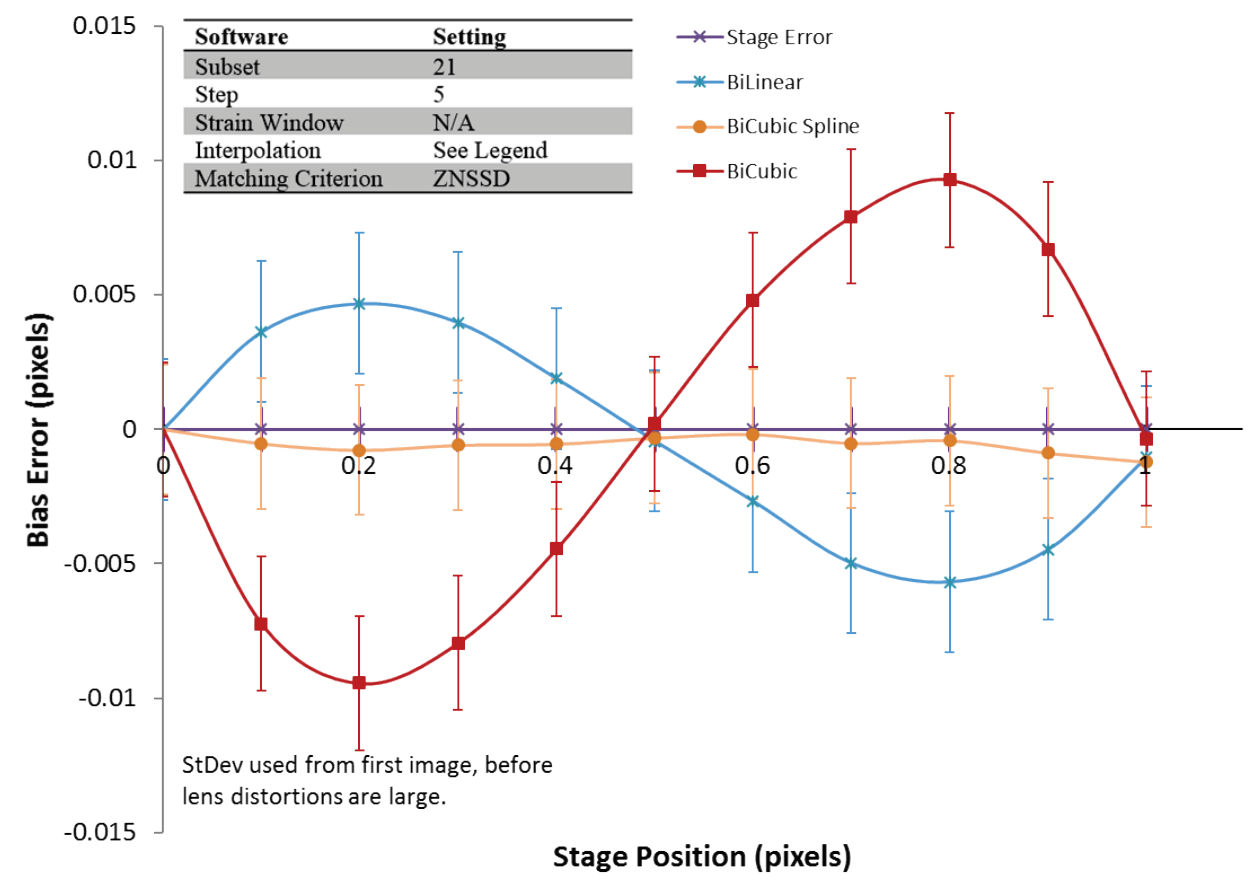

Figure 32. Prosilica subpixel shifting results showing three different interpolants. 


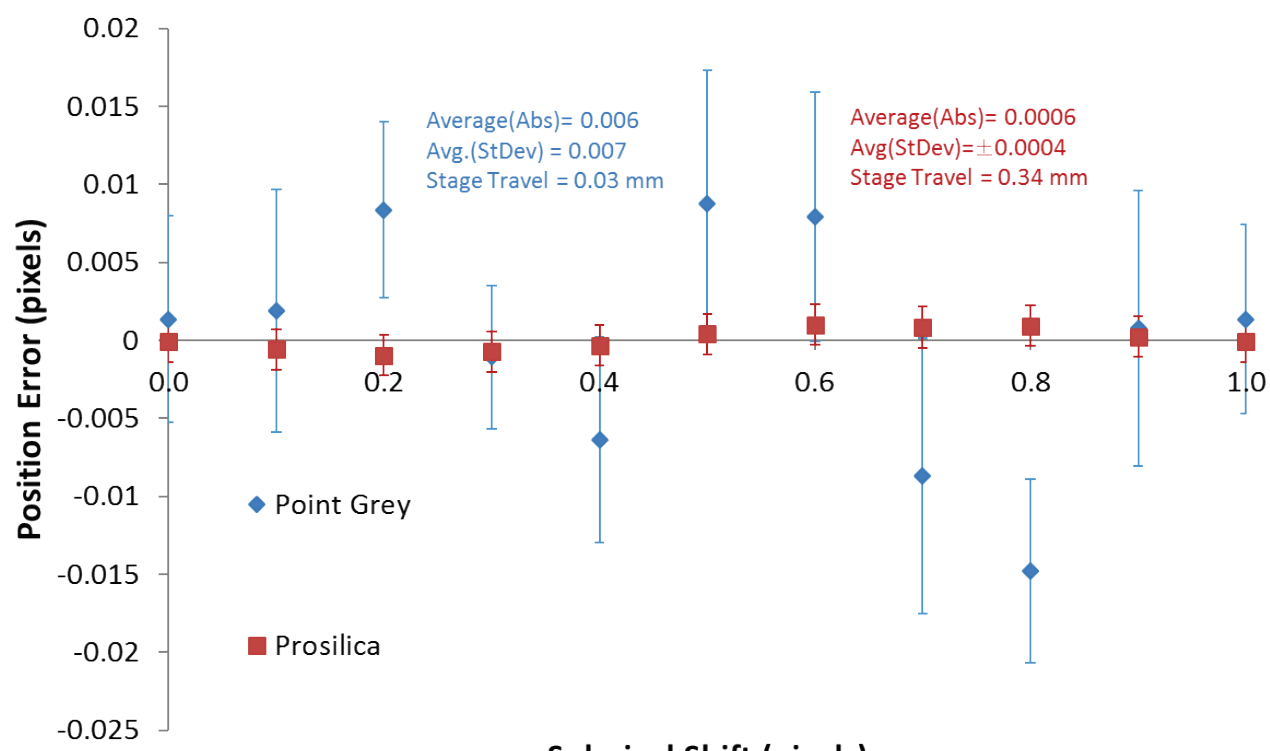

Subpixel Shift (pixels)

Figure 33. Comparison of a typical camera without binning and averaging versus the binned results. Note that the bias error for a typical camera setup is overwhelmed by the variance error (shown as error bars of \pm one standard deviation).

\section{A.9 Sample 17 (Out-of-plane Phase Shifted)}

Figure 34 shows the results from the DIC analysis of the out-of-plane shifted images. The $u$-displacement results shown are after fitting a best fit line to the $u$-displacement field and subtracting this best fit line from the raw $u$ displacements. This removes the large $u$-expansion seen and highlights the interpolant error. Each blue to red "sine" wave indicates a linear shift of 1-pixel over the period. The overall expansion of the image then is about 15 pixels in the $u$-direction (same results in $v$, although not shown). Calculation of the strain immediately shows the bias error, without need of removing the uniform expansion. Note the marked improvement in switching from a linear interpolant to a bi-cubic spline interpolant in the bias error. 


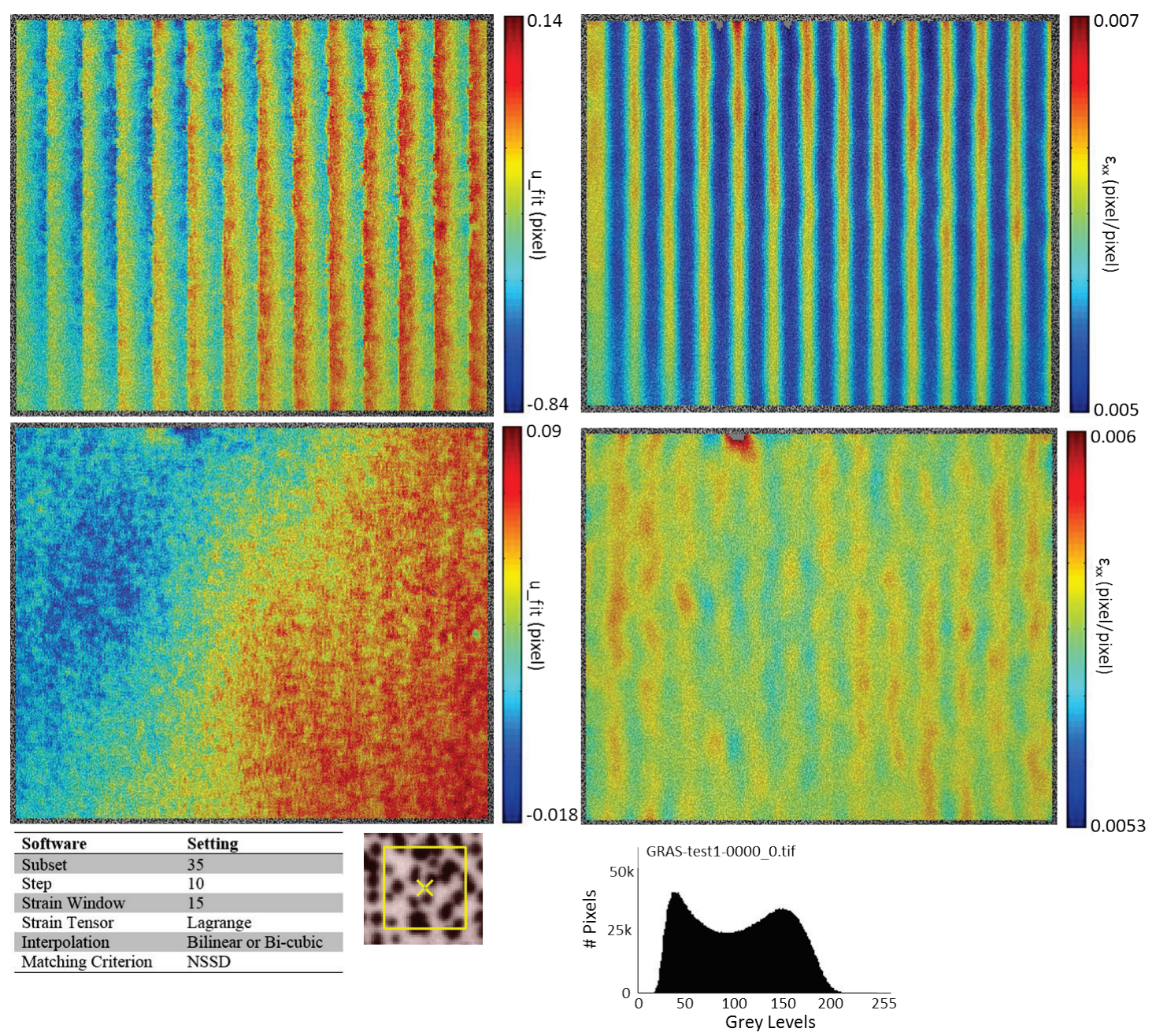

Figure 34. Sample 17 results for a bi-linear interpolant (top) showing both the udisplacement results (left) and the strain $\varepsilon_{\mathrm{xx}}$ results (right) and cubicspline interpolant (bottom). 


\section{Appendix B}

Appendix B contains some supplementary figures for Sample 14 that show the full-field results for all the codes.
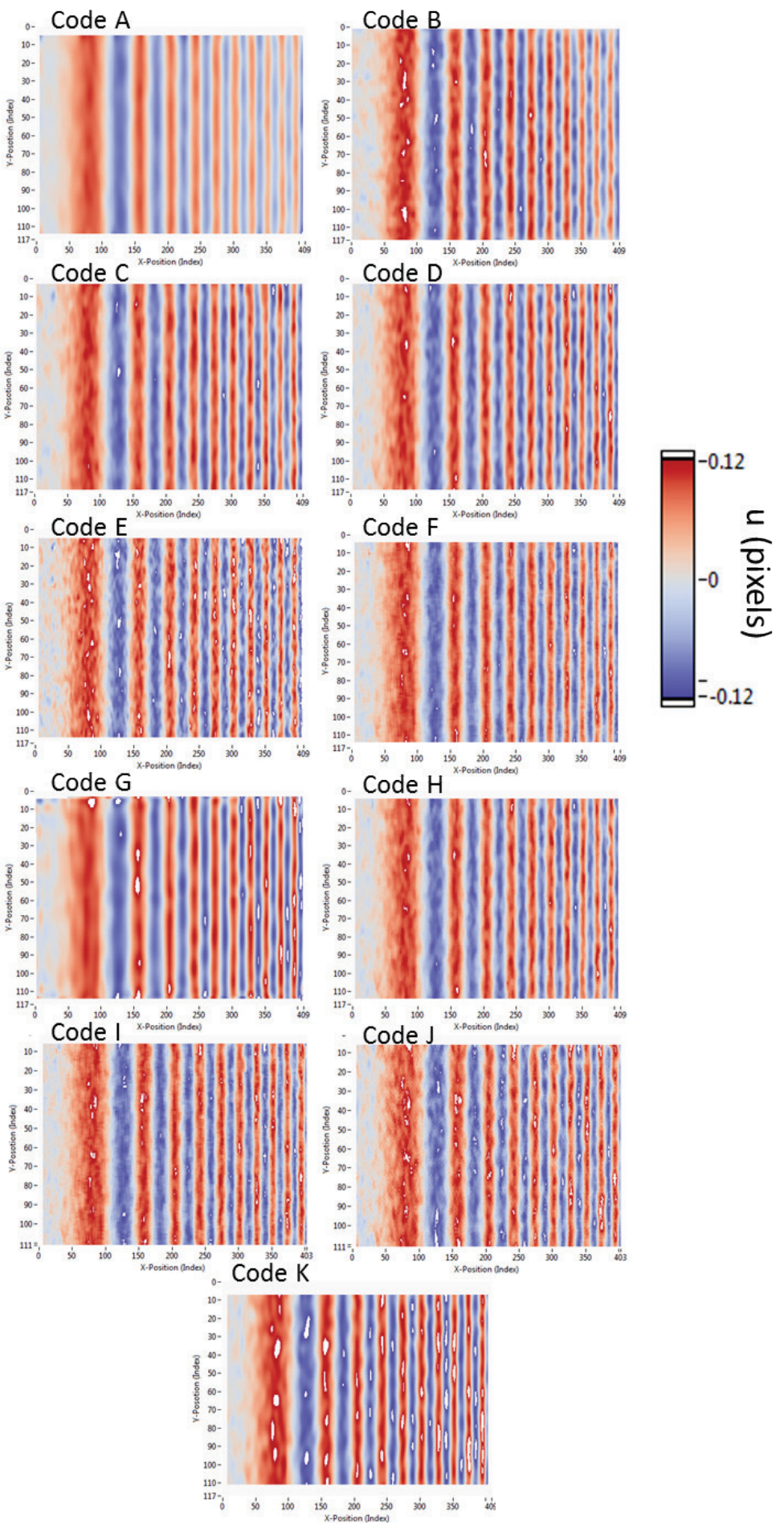

Figure 35. Image "Sample 14 L5 Amp0.1.tif" for all the codes as submitted by the participants and decoded and plotted by the LabVIEW code. Note some codes present results all the way to the edge of the FOV. White gaps in the displacement field indicate regions either above or below the scale limits rather than missing data. 

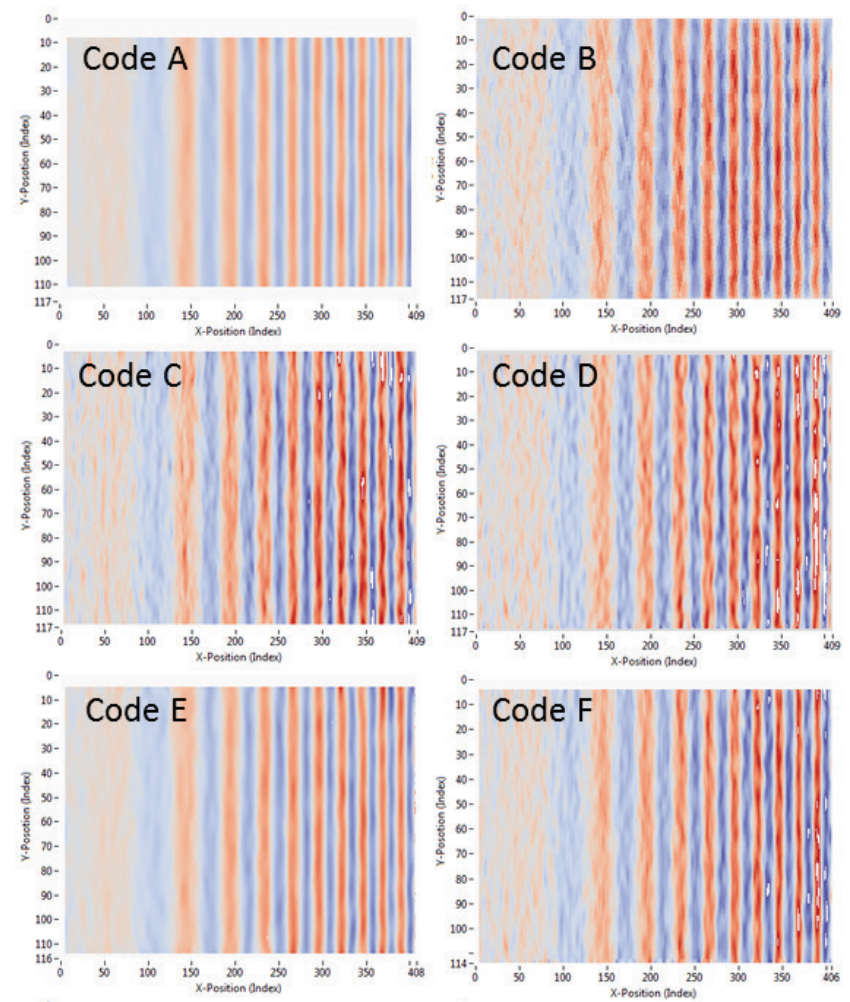

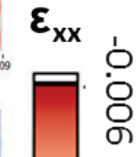
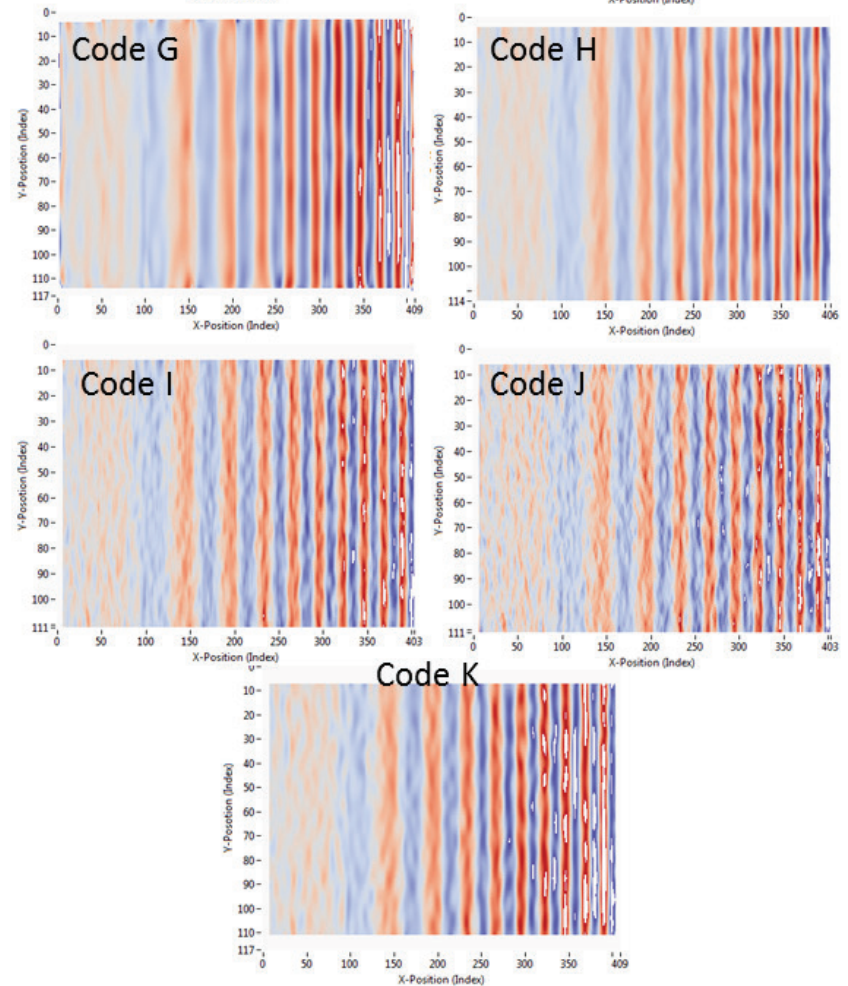

Figure 36. Sample 14 L5 full-field strain plots. Again, some bias errors can be seen, and a feel for the relationship between smoothing and noise is intuitively obvious. White gaps in the strain field indicate regions either above or below the scale limits rather than missing data. Note the small vertical strain banding on the left side in the zero strain region in all of the results. The reason for this is unknown, but ubiquitous. 

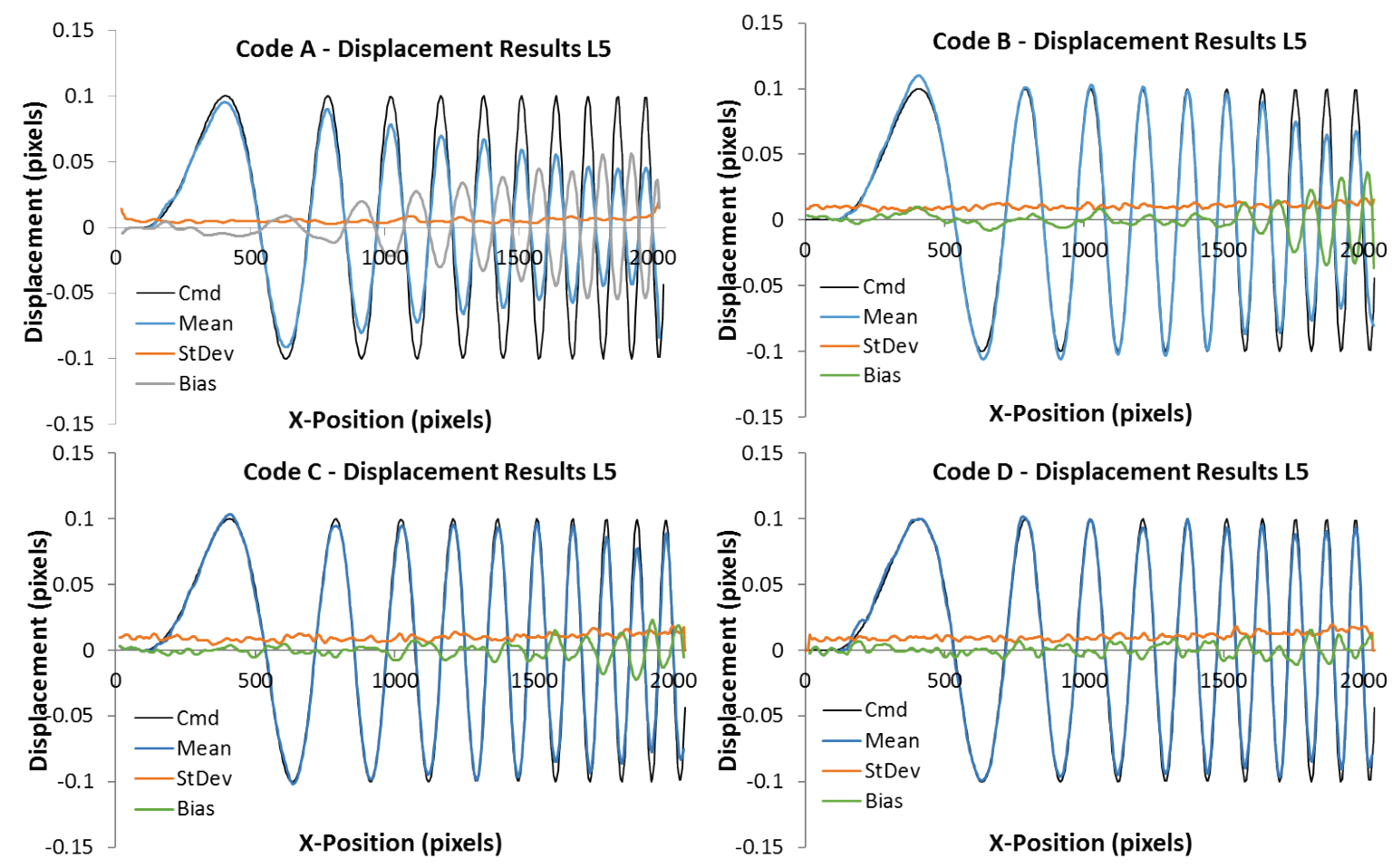

Figure 37. Displacement results for Sample 14 L5. Command, mean, bias and standard deviation curves are color coded in the plots. Typical results are shown. 